ARTICLE

\title{
Abraxas suppresses DNA end resection and limits break-induced replication by controlling SLX4/ MUS81 chromatin loading in response to TOP1 inhibitor-induced DNA damage
}

\author{
Xiao $\mathrm{Wu}^{1} \&$ Bin Wang (10 ${ }^{1,2}$
}

Although homologous recombination (HR) is indicated as a high-fidelity repair mechanism, break-induced replication (BIR), a subtype of $H R$, is a mutagenic mechanism that leads to chromosome rearrangements. It remains poorly understood how cells suppress mutagenic BIR. Trapping of Topoisomerase 1 by camptothecin (CPT) in a cleavage complex on the DNA can be transformed into single-ended double-strand breaks (seDSBs) upon DNA replication or colliding with transcriptional machinery. Here, we demonstrate a role of Abraxas in limiting seDSBs undergoing BIR-dependent mitotic DNA synthesis. Through counteracting K63-linked ubiquitin modification, Abraxas restricts SLX4/Mus81 recruitment to CPT damage sites for cleavage and subsequent resection processed by MRE11 endonuclease, CtIP, and DNA2/ BLM. Uncontrolled SLX4/MUS81 loading and excessive end resection due to Abraxasdeficiency leads to increased mitotic DNA synthesis via RAD52- and POLD3- dependent, RAD51-independent BIR and extensive chromosome aberrations. Our work implicates Abraxas/BRCA1-A complex as a critical regulator that restrains BIR for protection of genome stability.

\footnotetext{
${ }^{1}$ Department of Genetics, The University of Texas MD Anderson Cancer Center, Houston, Texas, USA. ${ }^{2}$ Genetics and Epigenetics Program, The MD

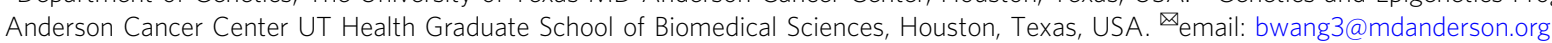


T opoisomerase I (TOP1) relaxes topological stress generated during DNA replication and transcription by temporarily forming a reversible TOP1-DNA cleavage complex (TOP1cc) nicking one strand and allowing rotation of the broken strand around the intact strand ${ }^{1,2}$. TOP1 inhibitor camptothecin $(\mathrm{CPT})$ and its clinical derivatives traps TOP1ccs at the enzyme-DNA interface forming protein-DNA crosslinks blocking DNA replication fork progression and ongoing transcription ${ }^{3}$. Replication forks colliding with the stabilized TOP1ccs induce replication-associated DNA double-strand breaks (DSBs). These are single-ended DSBs (seDSBs) caused by either replication runoff or cleavage of stalled replication forks by MUS81 $1^{4}$. Poisoning of TOP1 by CPT also induces accumulation of unscheduled Rloop, a three-stranded structure formed during transcription, which contain DNA-RNA hybrids and single-stranded displaced non-template DNA ${ }^{5-10}$. R loops induced by stalled Top1ccs lead to generation of transcription-dependent $\mathrm{DSBs}^{11}$. R-loopimpeded replication forks can be sensed and cleaved by MUS81 leading to DSBs ${ }^{12}$.

Two major pathways, homologous recombination (HR) and nonhomologous end joining (NHEJ) are involved in DSBs repair ${ }^{13}$. HR begins through DNA end resection that is initiated by MRE11-RAD50-NBS1 complex and CtIP through MRE11 endonuclease activity creating an adjacent DNA nick followed by nucleolytic processes by EXO1, DNA2 nucleases, and MRE11 exonuclease activity in both directions from the nick ${ }^{14}$. Compared to two-ended DSBs, seDSBs lack another DNA end to be ligated to for end joining, and thus are preferentially repaired by $\mathrm{HR}^{13}$. In budding yeast, it has been established that seDSBs repair undergo break-induced replication (BIR), a type of $\mathrm{HR}$ process that involves extensive $5^{\prime}$ to $3^{\prime}$-end resection to generate a $3^{\prime}$-singlestranded DNA (ssDNA) end that is bound by RPA, RAD52mediated strand invasion and formation of displacement loop (Dloop) intermediates and POLD3-dependent DNA synthesis ${ }^{15-17}$. In human cells, MUS81-mediated cleavage of collapsed replication forks at common fragile sites (CFS) initiates mitotic DNA synthesis (MiDAS) via BIR mechanism that depends on RAD52 and POLD3 but not on RAD51 ${ }^{18,19}$. BIR also occurs in response to oncogene overexpression, re-replication, or telomere erosion ${ }^{17,20}$. Although HR is indicated as a high-fidelity repair mechanism, BIR is a mutagenic mechanism that leads to chromosome rearrangements. The mechanism of resection to generate $3^{\prime}$-ssDNA and regulation of BIR has been extensively studied in yeast but still remains poorly understood in mammalian cells.

Abraxas interacts with BRCA1, forming a BRCA1-A complex with additional components Rap80, BRE, NBA1, and BRCC $36^{21}$. Abraxas is the central adapter protein of the BRCA1-A complex mediating the interaction of BRCA1 with the rest of the components ${ }^{21-25}$. Abraxas/BRCA1-A complex is recruited to DSBs in an Ataxia-Telangiectasia Mutated (ATM)-dependent signaling cascade that involves ubiquitin lysine 63 (K63)-linked modification on damaged chromatin catalyzed by E3 ligases RNF8/RNF168 and a K63-linkage-specific E2 conjugating enzyme $\mathrm{UBC} 13^{26,27}$. It has been shown that Abraxas and the formation of the BRCA1-A complex facilitates the deubiquitinating (DUB) activity of BRCC36 to disassemble K63-linked ubiquitin chain ${ }^{28,29}$. Despite the functional importance of Abraxas in recruitment of BRCA1 and tumor suppression ${ }^{21-24,30,31}$, the role of Abraxas in DNA repair still needs to be defined. In addition, the functional importance of Abraxas/BRCA1-A complex as a DUB complex is not clear.

BRCA1-A complex have been indicated a role in suppressing DSB resection, as defects in Rap80 and several other components of the BRCA1-A complex are linked to increased resection and appear to increase HR efficiency using a reporter assay ${ }^{32-34}$. It is intriguing how the Abraxas/BRCA1-A complex suppresses DNA end resection and $\mathrm{HR}$, while BRCA1 plays a promoting role in facilitating DNA end resection and HR.

Here we found that Abraxas inhibits excessive DNA end resection at CPT-induced seDSB sites by regulating K63-linked ubiquitin modification-dependent SLX4/MUS81 chromatin loading and subsequent cleavage of replication-associated DSBs. In the absence of Abraxas, increased K63-linked polyubiquitination led to enrichment of SLX4/MUS81 on damaged chromatin, causing excessive cleavage of CPT-induced stalled replication forks and R loops. DNA ends of cleaved replication-associated DSBs are further processed by MRE11 endonuclease, CTIP, and DNA2/BLM nucleases, generating excessive ssDNA that leads to MiDAS via BIR mechanism and chromosome aberrations. Our results demonstrate a role of Abraxas in regulating replication-associated seDSBs repair through inhibiting DNA synthesis-dependent BIR to safeguard genome stability.

\section{Results}

Abraxas limits DNA end resection of replication-associated DSBs. We found that when treated with CPT, Abraxas knockout (KO) U2OS cells or Abraxas null (-/-) mouse embryonic fibroblast (MEF) cells displayed increased phosphorylation of RPA32 S4/8 (pRPA), a surrogate marker of ssDNA accumulation and DNA end resection, upon treatment and at the indicated times after release into fresh media when compared to the control cells (Fig. 1a and Supplementary Fig. 1a). Total RPA32 level was not changed in Abraxas-deficient cells (Supplementary Fig. 1b). Immunofluorescence staining (IF) also showed increased staining of pRPA in Abraxas KO cells upon CPT treatment (Fig. 1b). Importantly, complementation with expression of hemagglutinin (HA)-tagged Abraxas in KO cells reduced the increased pRPA levels (Supplementary Fig. 1b). The increased pRPA level is correlated with increased ssDNA in Abraxas KO cells and Abraxas-/- MEFs detected by native Bromodeoxyuridine (BrdU) labeling and detection (Fig. 1c, d). To monitor DNA end resection, we carried out single-molecule analysis of resection tracks (SMART) to directly visualize resection at breaks ${ }^{35}$. It is apparent that Abraxas KO cells showed much increased resected BrdU-labeled ssDNA track length in response to CPT damage (Fig. 1e and Supplementary Fig. 1c). These data indicate that, compared to the control, there is increased DNA end resection and ssDNA generation in Abraxas-deficient cells.

CPT-induced stabilized Toplccs can readily be converted into seDSBs as replication forks collide with the stabilized Top1ccs ${ }^{4}$. To determine whether increased resection in Abraxas-deficient cells is associated with replication, we used aphidicolin (APH), an inhibitor of DNA polymerase to inhibit DNA synthesis before the treatment of cells with CPT. We found that inhibition of replication by APH completely abolished the increased pRPA levels in response to CPT in Abraxas KO U2OS cells and Abraxas-null MEFs, indicating that Abraxas inhibits DNA end resection at collapsed replication forks (Fig. 1f).

We then examined whether Abraxas localizes to CPT-induced DNA damage sites. Upon CPT treatment, Abraxas forms CPTinduced DNA damage foci using IF (Fig. 1g). In cells treated with CPT and pulse-labeled with EdU, Abraxas could be detected at DNA damage sites (marked by $\gamma \mathrm{H} 2 \mathrm{AX}$ ), which overlaps with newly synthesized DNA at replication forks (labeled by EdU), indicating that Abraxas is recruited to replication-associated DSBs (Fig. 1g).

In comparison, upon hydroxyurea (HU) treatment, the amount of ssDNA and pRPA levels were similar in Abraxasdeficient and control cells (Supplementary Fig. 1d, e), suggesting that stalled replication fork itself does not lead to increased resection in Abraxas-deficient cells. This is consistent with our 

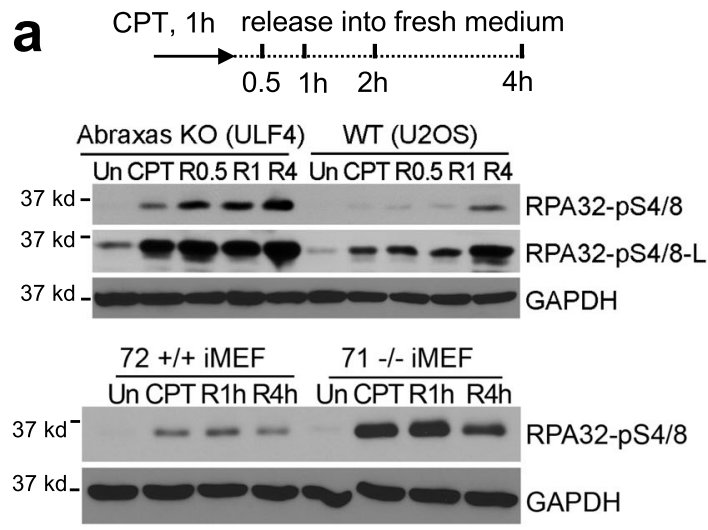

C

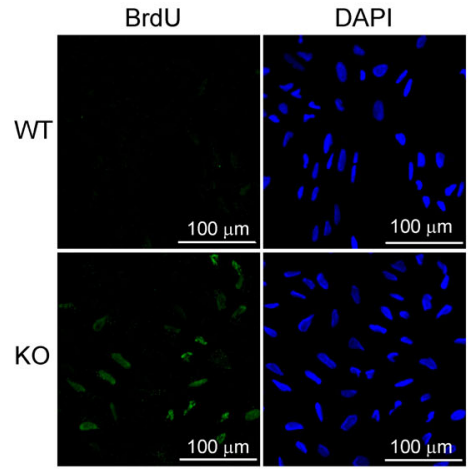

e


f


previous findings that Abraxas does not play a role in responding to replication stress induced by $\mathrm{HU}$ at stalled replication forks for fork protection or restart of stalled replication forks $\mathrm{s}^{30}$. Treatment with Mitomycin C (MMC) or poly (ADP-ribose) polymerase inhibitor, which forms DNA crosslink or protein-DNA crosslink that impedes DNA replication, however, led to elevated pRPA levels in Abraxas KO cells when compared to the control b

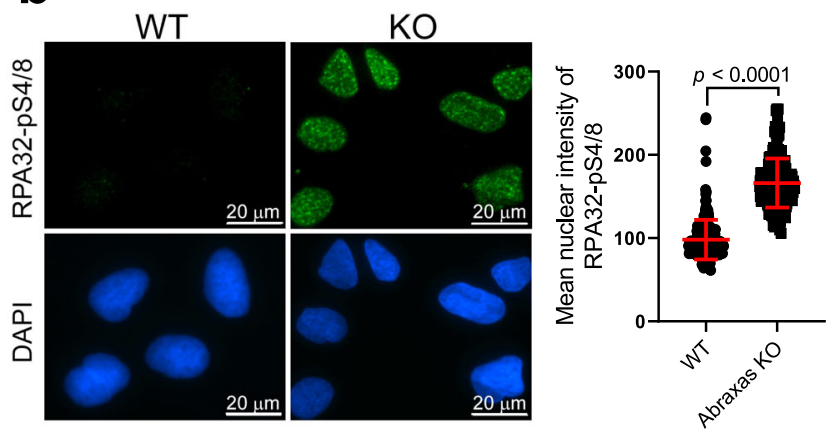

d


g

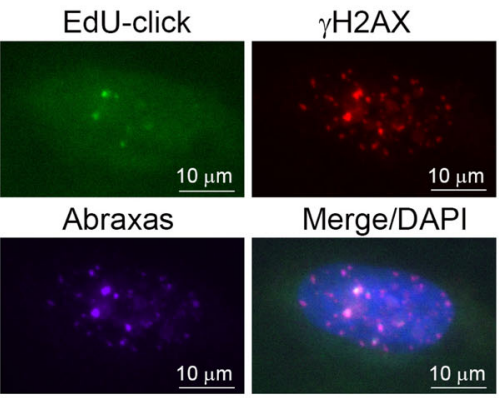

h

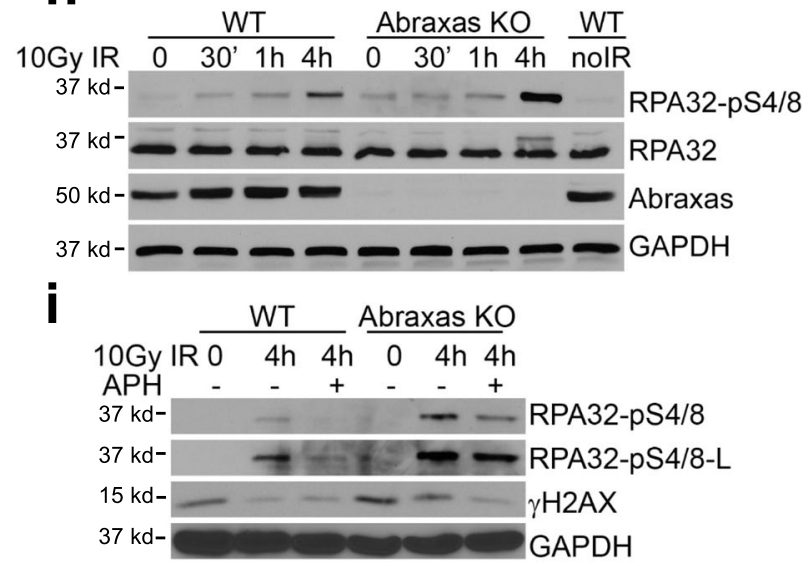

(Supplementary Fig. 1f, g). When treated with ionizing radiation (IR), compared to the control, Abraxas KO cells did not show elevated pRPA levels until a later time point, at $4 \mathrm{~h}$ after treatment, suggesting that Abraxas does not regulate the immediate processing of IR-induced two-ended DSB DNA ends, but involved in regulating resection at later steps during the repair of IR-induced DSBs (Fig. 1h). Interestingly, the increased pRPA 
Fig. 1 Abraxas limits DNA end resection of replication-associated DSBs. a Increased RPA32-pS4/8 levels in Abraxas knockout (KO) U2OS and Abraxas - / - MEF cells in response to CPT. Cells were untreated (Un), treated with $1 \mu \mathrm{M} \mathrm{CPT} \mathrm{for} 1 \mathrm{~h}$, released into fresh medium, and collected at indicated times (R0.5, R1, R4). b Immunofluorescence staining of RPA32-pS4/8 in WT and Abraxas KO U2OS cells treated with $1 \mu \mathrm{M} \mathrm{CPT} \mathrm{for} 1 \mathrm{~h}$. Cells were preextracted with $0.2 \%$ Triton X-100 before fixation. Nuclear intensity of RPA32-pS4/8 was quantified by ImageJ, shown as mean value \pm SD for WT ( $n=$ 244), KO ( $n=187)$ cells examined. Two-tailed unpaired $t$-test was used for statistical analysis. c Increased ssDNA accumulation in Abraxas KO U2OS cells with CPT treatment as detected by native BrdU immunofluorescence staining. Cells were labeled with BrdU for $36 \mathrm{~h}$ before being treated with $1 \mu \mathrm{M}$ CPT for $1 \mathrm{~h}$. Percentage of BrdU+ cells were plotted as mean value \pm SD for WT ( $n=24$ independent image areas with total 960 cells), KO ( $n=17$ independent image areas with total 960 cells). BrdU nuclear intensity were plotted as mean value \pm SD for WT $(n=960)$, KO $(n=960)$ cells examined. Two-tailed unpaired $t$-test was used for statistical analysis. Red dots represent BrdU nuclear intensity values higher than 40. d Increased ssDNA accumulation in Abraxas $-/-$ MEF cells in response to CPT $(1 \mu \mathrm{M}, 1 \mathrm{~h})$ detected by native BrdU immunofluorescence staining. BrdU intensity was measured using Image and plotted as mean value \pm SD for Abraxas $+/+(72+/+, n=382)$ or $-/-(71-/-, n=216)$ cells examined. Two-tailed unpaired $t$-test was used for statistical analysis. Red dots represent BrdU nuclear intensity values higher than 100. e Increased resection in Abraxas KO cells treated with CPT. Cells labeled with BrdU for $24 \mathrm{~h}$ were treated with CPT $(1 \mu \mathrm{M}, 1 \mathrm{~h})$. DNA fibers were stained with BrdU under native condition. Total fibers were also stained with YOYO-1 and shown in Supplementary Fig. 1c. The length of BrdU-stained DNA fiber was measured by ImageJ and plotted as mean value \pm SD for WT ( $n=$ 246) and KO $(n=371)$ DNA fibers. Two-tailed unpaired $t$-test was used for statistical analysis. $\mathbf{f}$ Inhibition of DNA replication by aphidicolin (APH) abolished RPA hyperphosphorylation in Abraxas-deficient cells. APH (0.1 $\mu \mathrm{M})$ was added to medium 15 min before cells were treated with $1 \mu \mathrm{M}$ CPT. Cells were then released into fresh medium containing APH and collected at indicated times. $\mathbf{g}$ Abraxas accumulates at replication-associated DSBs. Cells were treated with CPT $(1 \mu \mathrm{M}, 1 \mathrm{~h})$, pulsed labeled with EdU for 15 min before EdU-click and IF staining with antibodies to Abraxas and $\gamma \mathrm{H} 2 \mathrm{AX}$. $\mathbf{h}$ Elevated RPA32pS4/8 levels in Abraxas KO cells at $4 \mathrm{~h}$ after IR treatment. WT and KO cells were treated with $10 \mathrm{~Gy}$ IR and collected at 0, 30 min, $1 \mathrm{~h}$, and $4 \mathrm{~h}$. Western blottings were performed using indicated antibodies. i Inhibition of replication by APH reduced RPA hyperphosphorylation upon $10 \mathrm{~Gy}$ IR. APH was added to medium 15 min before IR. RPA32-pS4/8 levels were compared between WT and Abraxas KO cells at $4 \mathrm{~h}$ after $10 \mathrm{~Gy}$ IR without or with $0.1 \mu \mathrm{M}$ APH incubation. Student's t-test was used for statistical analysis of b-d. See also Supplementary Fig. 1.

levels in Abraxas KO cells at the later time point ( $4 \mathrm{~h}$ ) after IR treatment also depend on replication, as addition of APH to cells 15 min before the treatment and during the incubation decreased the elevated level of pRPA (Fig. 1i).

Abraxas suppresses R-loop accumulation and R-loopassociated DNA end resection. As TOP1 plays important roles in transcription initiation and elongation, persistent TOP1ccs can induce transcription block and formation of R-loop, a transcriptional intermediate structure with RNA:DNA hybrid and displaced $\mathrm{ssDNA}^{5-7}$. We tested CPT-induced R-loop formation in Abraxas-deficient cells. Using S9.6 antibody to detect R-loop levels in a dot blot assay, we found that, compared to the control, Abraxas $\mathrm{KO}$ cells showed a greater increase in $\mathrm{R}$-loop levels with CPT treatment and at $1 \mathrm{~h}$ after release from CPT (Fig. 2a). IF staining also revealed that $\$ 9.6$ nuclear signal significantly increased in Abraxas $\mathrm{KO}$ cells in response to CPT treatment (Fig. 2b, c). These data indicate that Abraxas deficiency leads to $\mathrm{R}$-loop accumulation in response to $\mathrm{CPT}$ treatment. To determine whether R-loop formation contributes to increased resection observed in Abraxas-deficient cells, we overexpressed RNaseH1, which degrades the RNA hybridized with DNA in the R-loop structure, in Abraxas $\mathrm{KO}$ cells ${ }^{36}$. Overexpression of $\mathrm{RNaseH} 1$ reduced the elevated pRPA upon CPT treatment to the control level (Fig. 2d). It suggests that the increased resection marked by elevated pRPA in Abraxas-deficient cells is associated with R-loop processing upon treatment of CPT.

Inhibition of transcription using transcription inhibitor 5, 6dichloro-1- $\beta$-d-ribofuranosylbenzimidazole (DRB) also decreased the elevated pRPA, suggesting that Abraxas plays a role in inhibiting resection at places when transcription machinery clashed into TOP1cc sites (Fig. 2e). Abraxas deficiency or treatment of DRB did not have much effect on cell cycle distribution, suggesting that the effect of DRB on resection is not due to a change of cell cycle distribution (Supplementary Fig. 2a). Another transcription inhibitor $\alpha$-amanitin treatment was also able to reduce pRPA levels in Abraxas KO cells after CPT treatment (Supplementary Fig. 2b).

Together, Abraxas suppresses CPT-induced R-loop accumulation and DNA end resection at trapped TOP1cc sites associated with interference of transcriptional machineries.
MUS81 overloading on chromatin in Abraxas-deficient cells leads to increased resection. The structure-specific endonuclease MUS81 is involved in the cleavage of TOP1cc-stalled replication forks and R-loop-impeded replication forks ${ }^{4,12}$. We found that chromatin-bound MUS81 level was higher in Abraxas KO cells when compared to that of the control. IF staining of chromatinbound MUS81 for cells pre-extracted with Triton X-100 before fixation showed enriched MUS81 intensity in Abraxas KO cells upon CPT treatment (Fig. 3a and Supplementary Fig. 3a). Chromatin fractionation analysis also showed that MUS81 loading on chromatin were greater in Abraxas KO cells (Fig. 3b). Importantly, knocking down MUS81 largely reduced elevated pRPA in Abraxas KO cells treated with CPT, indicating that the extensive resection of CPT-induced collapsed replication forks in Abraxas KO cells depends on MUS81 (Fig. 3c).

We then tested whether MUS81 overloading results in uncontrolled MUS81-mediated cleavage leading to increased DSBs. Using $\gamma \mathrm{H} 2 \mathrm{AX}$ as a DSB marker, we found that the percentage of cells containing $\gamma \mathrm{H} 2 \mathrm{AX}$ foci significantly increased (Fig. 3d and Supplementary Fig. 3b) in Abraxas KO cells treated with CPT. Neutral comet assay also showed that Abraxas KO cells displayed increased DSBs when compared to the control upon treatment of CPT (Fig. 3e). Importantly, knocking down MUS81 decreased CPT-induced DSBs detected by neutral comet assay and $\gamma \mathrm{H} 2 \mathrm{AX}$ levels in Abraxas $\mathrm{KO}$ cells (Fig. 3f, $\mathrm{g}$ and Supplementary Fig. 3c). EdU pulse labeling and IF with $\gamma \mathrm{H} 2 \mathrm{AX}$ and pRPA showed that replication-associated DSBs and resection marked by damage foci with colocalization of EdU, $\gamma \mathrm{H} 2 \mathrm{AX}$, and pRPA increased in Abraxas $\mathrm{KO}$ cells treated with CPT (Supplementary Fig. 3d). Thus, Abraxas deficiency results in uncontrolled MUS81-mediated cleavage, leading to increased DSBs that are subjected to subsequent resection.

Increased resection in Abraxas-deficient cells is independent of fork reversal but requires MRE11 endonuclease, CtIP, DNA2/ BLM. MUS81 cleaves a number of DNA replication fork-like intermediates including both stalled and reversed replication fork $^{37,38}$. To understand the effect of replication fork reversal on increased resection in Abraxas-deficient cells, we tested pRPA levels in cells depleted of SMARCAL1, ZRANB3 or HLTF, factors that are needed for replication fork reversal ${ }^{39-44}$. Depletion of 
a
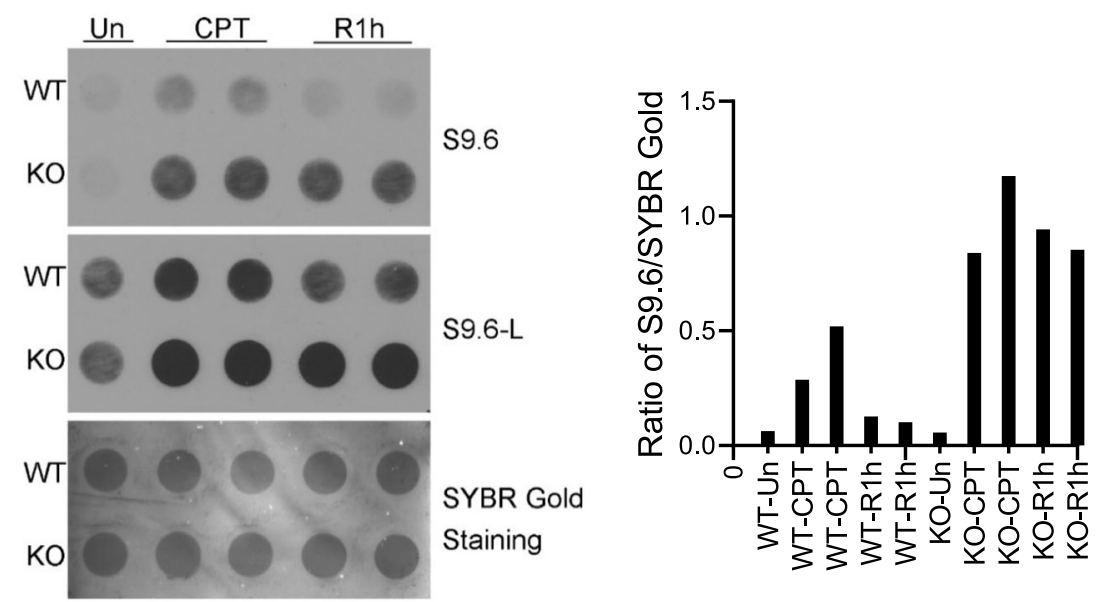

b
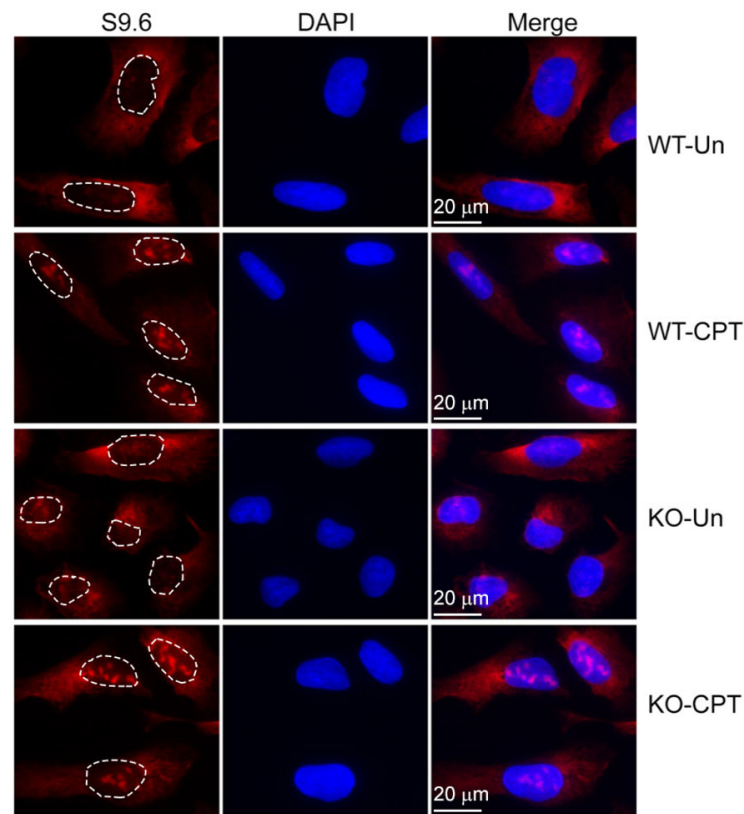

C
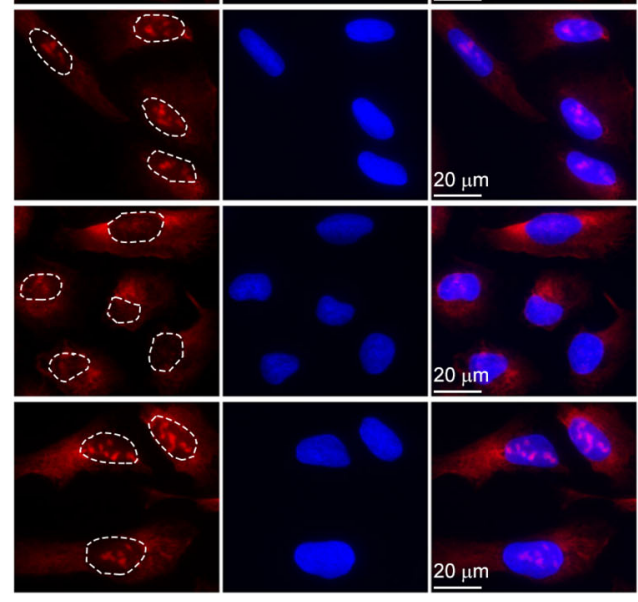

d
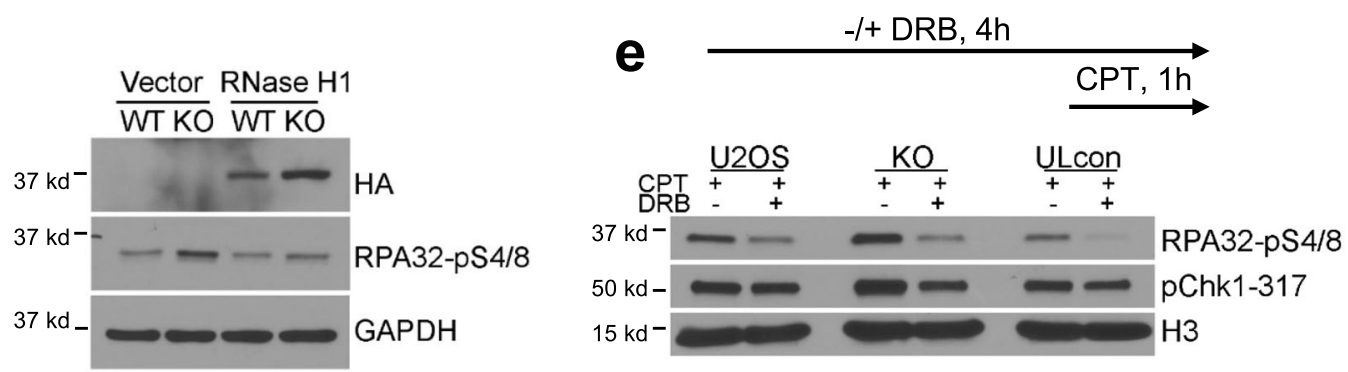

Fig. 2 Abraxas suppresses R-loop accumulation and R-loop-associated DNA end resection. a Increased R loops in Abraxas KO cells upon CPT treatment. Dot blot using S9.6 antibody of DNA from cells untreated, treated with CPT $(1 \mu \mathrm{M}, 1 \mathrm{~h})$, or at $1 \mathrm{~h}$ after release into fresh medium (left panel). Total genomic DNA was extracted and used for R-loop detection. SYBR Gold staining of the same membrane served as a loading control. S9.6 intensity was measured by Image and normalized to loading control for quantification (right panel). b S9.6 immunofluorescence staining in WT and Abraxas KO cells. Cells untreated or treated with CPT $(1 \mu \mathrm{M}, 1 \mathrm{~h})$ were pre-extracted with $0.2 \%$ Triton X-100 before fixation. c S9.6 nuclear intensity was quantified by Image J software and plotted as mean \pm SD for WT-Un $(n=117)$, KO-Un $(n=133)$, WT-CPT $(n=194)$, and KO-CPT $(n=121)$ cells examined. One-way ANOVA Kruskal-Wallis test was used for statistical analysis. ${ }^{\star \star \star} p=0.0002,{ }^{\star \star \star \star} p<0.0001$. d RNaseH1 expression reduced RPA32-pS4/8 levels in Abraxas KO cells treated with CPT. Cells transfected with vector or HA-tagged RNaseH1 were treated with CPT. RNaseH1 expression was detected by HA antibody. e Addition of transcription inhibitor DRB reduced RPA32-pS4/8 levels in Abraxas KO cells. Then, $20 \mu \mathrm{M} 5$, 6-dichloro-1- $\beta$-d-ribofuranosylbenzimidazole (DRB) was added $3 \mathrm{~h}$ before CPT treatment $(1 \mu \mathrm{M}, 1 \mathrm{~h})$. See also Supplementary Fig. 2. 
a

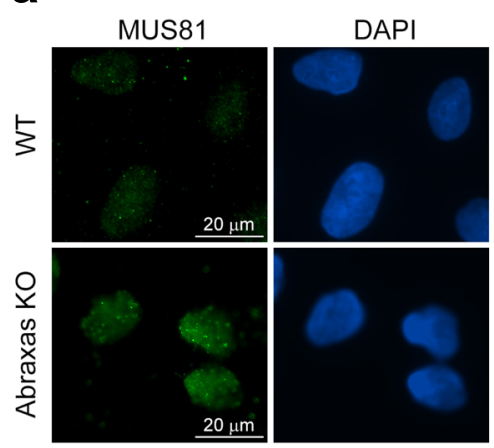

C



$\mathbf{f}$

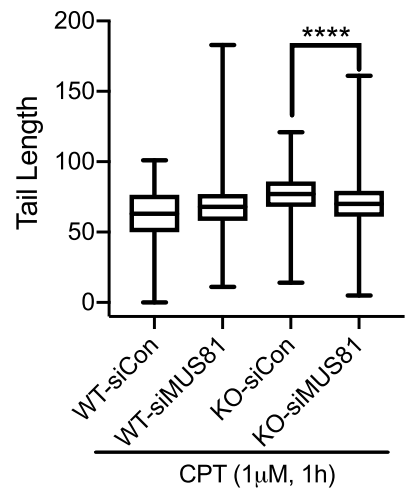

g b



d

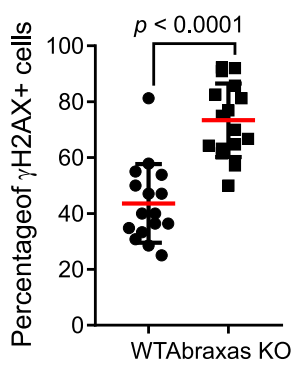

e

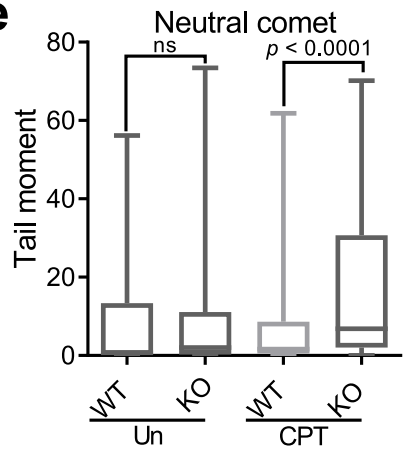

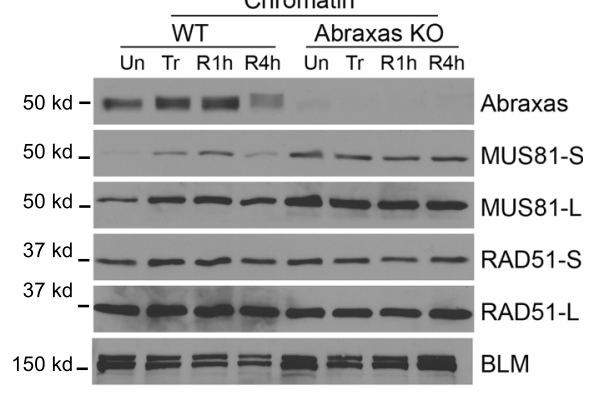

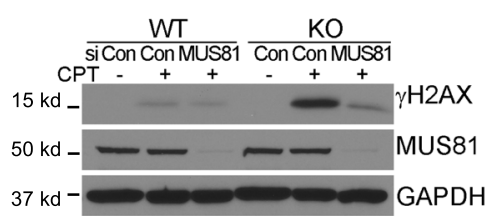

Fig. 3 MUS81 overloading on chromatin in Abraxas-deficient cells leads to increased resection. a Increased chromatin-bound MUS81 in Abraxas KO cells detected by immunofluorescence. Cells upon CPT treatment were pre-extracted with $0.2 \%$ Triton X-100 before fixation and stained with MUS81 antibody. Representative images of MUS81 staining (left panel) and quantification of MUS81 nuclear intensity measured by ImageJ (right panel) are shown as mean value \pm SD for WT-CPT $(n=142)$, KO-CPT $(n=168)$ cells examined. Two-tailed Student's $t$-test was used for statistical analysis. b Increased chromatin-bound MUS81 in Abraxas KO cells detected by cell fractionation and western blotting. Chromatin fraction of cells untreated (Un), treated with $1 \mu \mathrm{M} \mathrm{CPT}$ for $1 \mathrm{~h}(\mathrm{Tr})$, or released into fresh medium for $1 \mathrm{~h}$ (R1h) or $4 \mathrm{~h}$ (R4h) after treatment were prepared for western blottings. c Elevated RPA32-pS4/ 8 levels in Abraxas KO cells upon CPT treatment was reduced by MUS81 knockdown. Cells transfected with siRNAs were untreated or treated with $1 \mu \mathrm{M}$ CPT for $1 \mathrm{~h}$. $\mathbf{d}$ Quantification of $\gamma \mathrm{H} 2 \mathrm{AX}$ immunofluorescence staining in WT and Abraxas KO U2OS cells treated with CPT $(1 \mu \mathrm{M}, 1 \mathrm{~h})$. Percentage of cells containing more than $10 \gamma \mathrm{H} 2 \mathrm{AX}$ foci was quantified as mean value \pm SD for WT ( $n=16$ independent image areas with total 319 cells) and KO ( $n=16$ independent image areas with total 401 cells). Two-tailed $t$-test was used for statistical analysis. e Increased DSBs in Abraxas KO cells treated with CPT $(1 \mu \mathrm{M}, 1 \mathrm{~h})$ as detected by neutral comet assay. Tail moments were measured by open comet software and plotted as box plot. Center line indicates median, box bounds indicate first and third quartiles, whiskers indicated maximum and minimum for WT-Un $(n=274)$, KO-Un ( $n=152)$, WT-CPT ( $n=$ $383)$, and KO-CPT $(n=249)$ cells examined. One-way ANOVA Kruskal-Wallis test was used for statistical analysis. $\mathbf{f}$ MUS81 knockdown reduced DSBs accumulation in Abraxas KO cells upon CPT treatment as detected by neutral comet assay. Tail length was measured by open comet software and plotted as box plot. Center line indicates median, box bounds indicate first and third quartiles, whiskers indicated maximum and minimum for WT-siCon ( $n=161$ ), WT-siMUS81 $(n=271)$, KO-siCon $(n=180)$, and KO-siMUS81 $(n=286)$ cells examined. One-way ANOVA Kruskal-Wallis test was used for statistical analysis. ${ }^{\star \star \star \star} p<0.0001$. g MUS81 knockdown reduced $\gamma \mathrm{H} 2 \mathrm{AX}$ levels in Abraxas KO cells upon CPT treatment. Cells transfected with indicated siRNAs were untreated or treated with $1 \mu \mathrm{M}$ CPT for $1 \mathrm{~h}$. See also Supplementary Fig. 3.

neither SMARCAL1 nor ZRANB3 rescued hyperphosphorylation of RPA in Abraxas KO cells treated with CPT (Fig. 4a and Supplementary Fig. 4a, b). Depletion of HLTF also did not have much effect on the hyperphosphorylation of RPA in Abraxas KO cells (Fig. 4b). These data indicate that fork reversal is not required for RPA hyperphosphorylation and excessive end resection in Abraxas-deficient cells.
We then investigated the nucleases involved in DSBs end resection. MRE11, together with CtIP, initiates DSB end resection by generating short end resections that can be further processed by two additional nuclease complexes containing either DNA2/ BLM or EXO1/BLM ${ }^{13,14}$. To determine whether these nucleases are involved in the excessive resection of replication-associated seDSBs in Abraxas-deficient cells, we assessed the effect of 
a

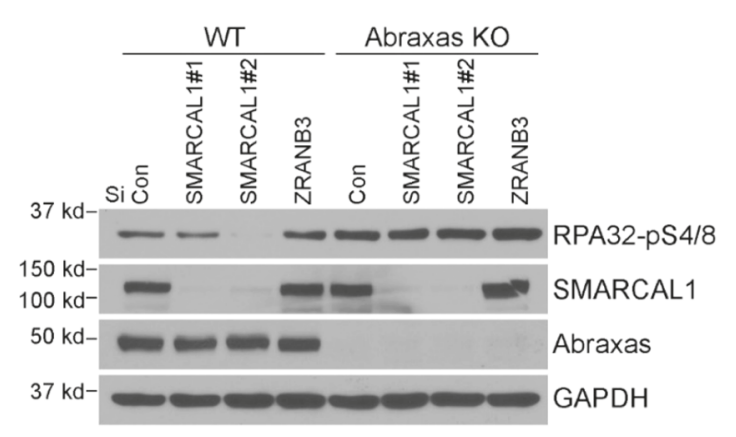

d

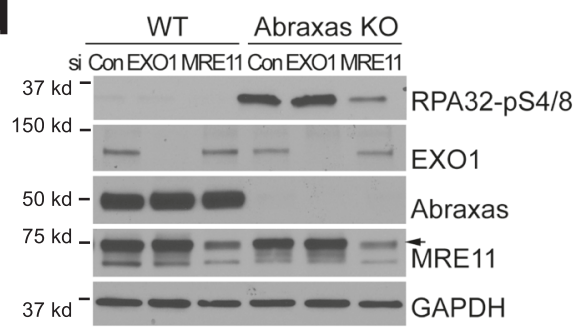

f

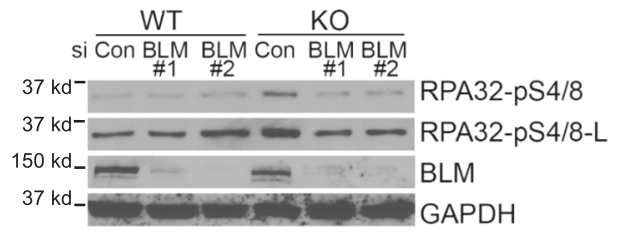

b
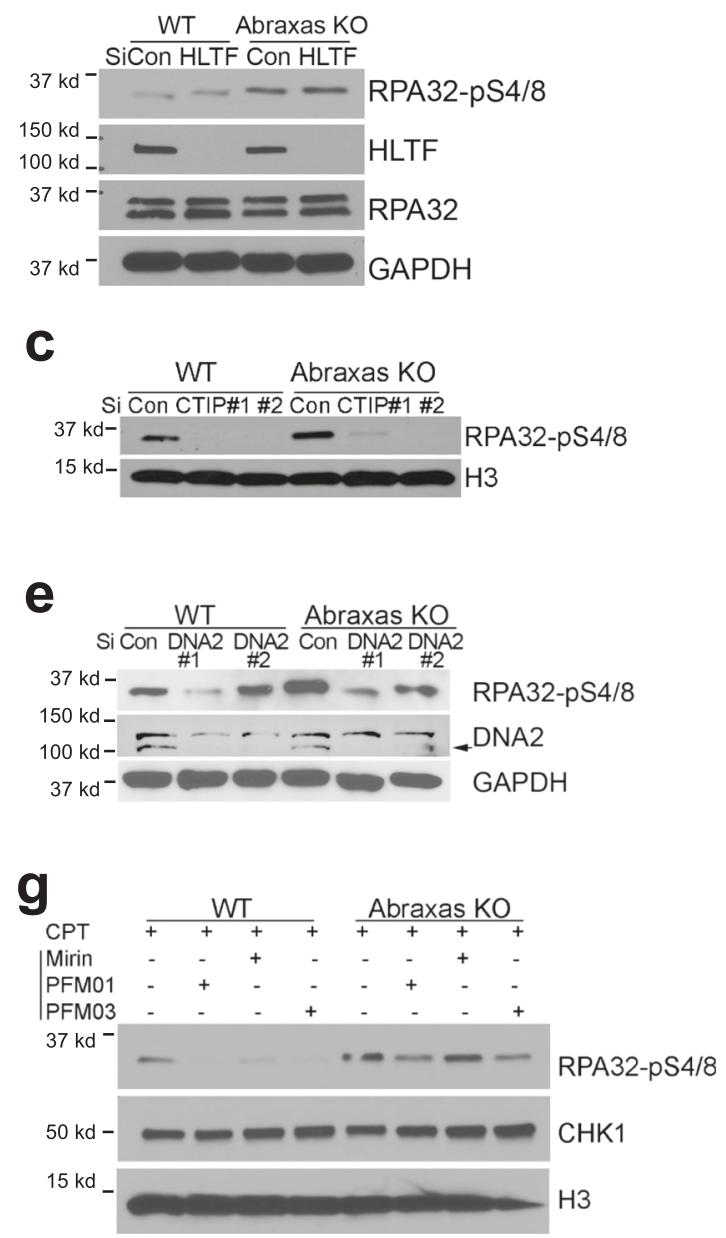

Fig. 4 Increased resection in Abraxas-deficient cells is independent of fork reversal but requires MRE11 endonuclease, CtIP, and DNA2/BLM. a SMARCAL1 and ZRANB3 depletion does not rescue CPT-induced hyperphosphorylation of RPA in Abraxas KO cells. Data of additional siRNAs to SMARCAL1 are in Supplementary 4a. b HLTF depletion does not rescue CPT-induced hyperphosphorylation of RPA in Abraxas KO cells. c Knocking down CtIP reduced the elevated RPA32-pS4/8 levels in Abraxas KO cells treated with CPT. CtIP knockdown efficiency is shown in Supplementary Fig. S4. d Knocking down MRE11 but not EXO1 reduced the elevated RPA32-pS4/8 levels in Abraxas KO cells treated with CPT. e Knocking down DNA2 reduced the elevated RPA32-pS4/8 levels in Abraxas KO cells treated with CPT. Data of additional siRNAs to DNA2 are in Supplementary Fig. 4a. f Knocking down BLM reduced the elevated RPA32-pS4/8 levels in Abraxas KO cells treated with CPT. $\mathbf{g}$ MRE11 endonuclease activity but not exonuclease activity is required for increased RPA32-pS4/8 levels in Abraxas KO cells upon CPT treatment. MRE11 endonuclease inhibitors PFM01 and PFM03 (100 $\mu$ M) or exonuclease inhibitor Mirin $(100 \mu \mathrm{M})$ was added $1 \mathrm{~h}$ before cells were treated with CPT $(1 \mu \mathrm{M}, 1 \mathrm{~h})$. CHK1 and H3 served as loading controls. See also Supplementary Fig. 4.

knockdown of each of these genes on pRPA levels in Abraxas KO cells treated with CPT. We found that knockdown of CtIP, MRE11, and DNA2/BLM, but not EXO1, reduced pRPA to the control level (Fig. 4c-f and Supplementary Fig. 4). Importantly, the cell cycle distribution was not drastically changed in Abraxas $\mathrm{KO}$ cells depleted of various nucleases, suggesting that the effect of depletion of CtIP, MRE11, or DNA2/BLM on resection was not due to a change of cell cycle distribution (Supplementary Fig. 4f). The Mre11 nuclease catalytic domain possesses both endo- and exo-nucleolytic activity, both of which are involved in end resection of two-ended DSBs. The endonuclease activity of MRE11 is required to initiate the resection and CtIP promotes the endonucleolytic activity of MRE11 ${ }^{45}$. We further asked whether it is the endonuclease or exonuclease activity of MRE11 that is involved in the extended resection using different MRE11 inhibitors targeting the endo- or exonuclease activity ${ }^{45}$. Inhibitors PFM01 and PFM03, which primarily block MRE11 endonuclease activity, reduced RPA phosphorylation, whereas Mirin, an
MRE11 exonuclease inhibitor, did not (Fig. 4g). These results indicate that MRE11 endonuclease activity is involved in the uncontrolled resection that occur due to Abraxas deficiency.

Together, the excessive resection of replication-associated seDSBs in Abraxas-deficient cells does not require fork reversal. It is also distinct from the resection of two-ended DSBs, requiring Mre11 endonuclease, CtIP, and DNA2/BLM, but not MRE11 exonuclease or EXO1.

Abraxas limits K63-linked ubiquitin-dependent SLX4/MUS81 recruitment to CPT damage sites. The scaffold protein SLX4 interacts with MUS81 and has been shown to stimulate MUS81 activity in vitro and coordinates the resolution of Holliday junctions ${ }^{46,47}$. We examined whether MUS81 overloading on chromatin is dependent on SLX4. Similar to MUS81, SLX4 level was also elevated on CPT-damaged chromatin in Abraxas KO cells (Fig. 5a lane 1 and 3). Knocking down SLX4 significantly 
a

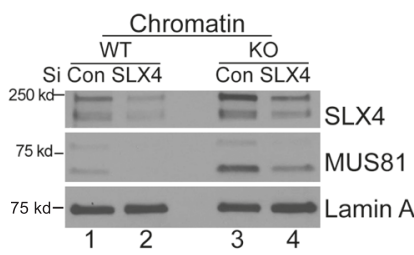

b

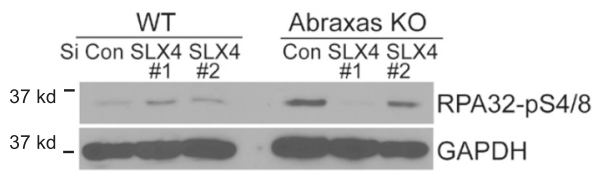

C

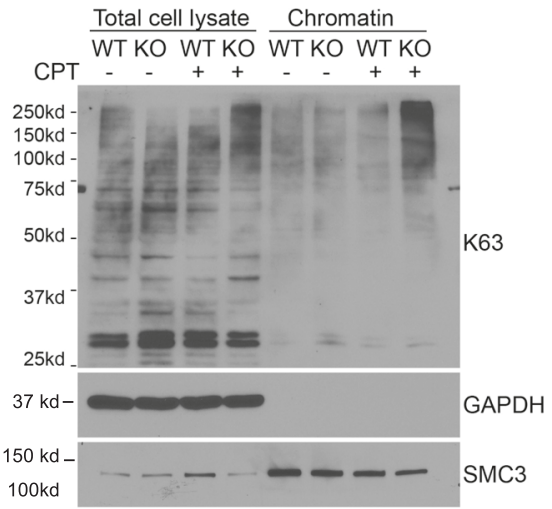

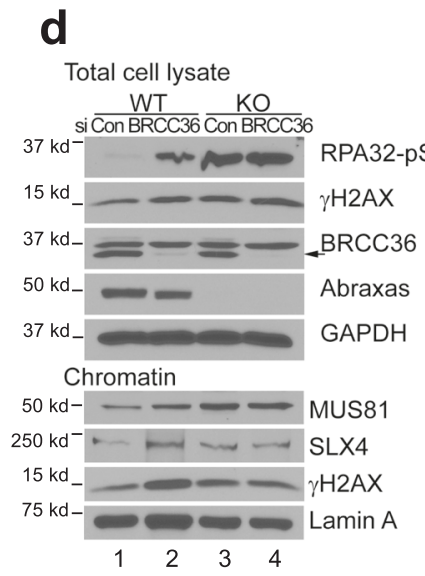

e Total cell lysate

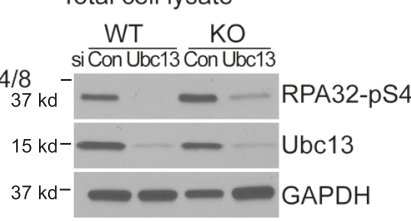

f

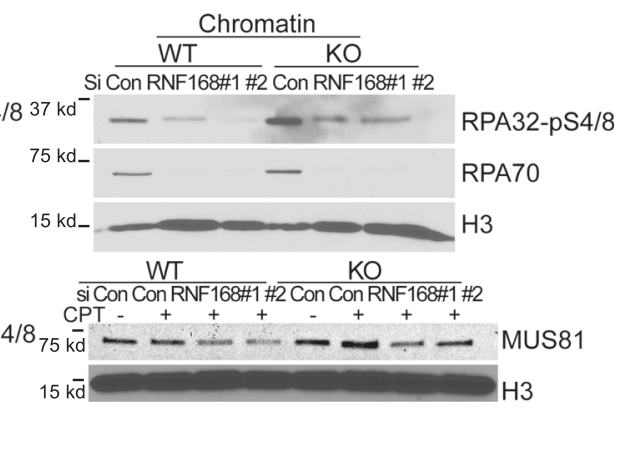

Fig. 5 Abraxas limits K63-linked ubiquitin-dependent SLX4/MUS81 recruitment to CPT damage sites. a Increased chromatin-bound MUS81 in Abraxas KO cells was reduced by SLX 4 knockdown. Cells transfected with indicated siRNAs were treated with $1 \mu \mathrm{M}$ CPT for $1 \mathrm{~h}$. Chromatin fraction was isolated for analysis by western blottings. b SLX4 knockdown reduced the elevated RPA32-pS4/8 levels in Abraxas KO treated with CPT. Total lysates were used for western blottings. c K63-linked polyubiquitination is increased in Abraxas KO U2OS cells treated with CPT. WT and Abraxas KO U2OS cells were untreated or treated with $1 \mu \mathrm{M} \mathrm{CPT}$ for $1 \mathrm{~h}$. Total cell lysates and chromatin fractions were prepared from samples for western blottings. K63-linked ubiquitination was detected by antibodies against K63-linked chain. $\mathbf{d}$ BRCC36 knockdown leads to increased RPA32-pS4/8 and overloading of SLX4/MUS81 to CPT-

damaged chromatin. Total cell lysates and chromatin fractions were prepared from cells transfected with indicated siRNAs and treated with CPT ( $1 \mu \mathrm{M}, 1$ h). e Ubc13 knockdown reduced elevated RPA32-pS4/8 levels and chromatin-bound MUS81 in Abraxas KO cells treated with CPT. f RNF168 knockdown reduced elevated RPA32-pS4/8 levels and chromatin-bound MUS81 in Abraxas KO cells treated with CPT. See also Supplementary Fig. 5.

reduced chromatin MUS81 levels (Fig. 5a lane 3 and 4, and Supplementary Fig. 5a) and reduced pRPA in Abraxas KO cells (Fig. 5b and Supplementary Fig. 5b). Therefore, SLX4 functions upstream of MUS81 in promoting MUS81 chromatin loading to CPT damage sites. In contrast, knockdown of CtIP or DNA2 decreased pRPA levels in Abraxas KO cells, but had little effect on the amount of MUS81 on CPT-damaged chromatin (Supplementary Fig. $5 \mathrm{c}$ ), consistent with the idea that these nucleases acting on resecting DSBs ends downstream of MUS81 cleavage.

SLX4 contains two tandem UBZ domains that bind to K63linked ubiquitin chains for the recruitment of the protein to crosslink DNA damage sites ${ }^{48-50}$. To confirm that SLX4 recruitment to CPT damage sites through its UBZ domain is involved, we tested whether UBZ domain is important for the increased pRPA levels in Abraxas KO cells. Re-introduction of HA-tagged wild-type (WT) SLX4 gene, but not UBZ mutant, to Abraxas KO cells depleted of SLX4 restored the hyperphosphorylation of RPA, indicating that the UBZ domain of SLX4 is critical in the increased resection in Abraxas KO cells (Supplementary Fig. 5d).

We then investigated whether there is an increase of K63linked ubiquitination on CPT-damaged chromatin in Abraxasdeficient cells. We found that, compared to WT control, K63linked ubiquitin conjugates on CPT-damaged chromatin are significantly increased in Abraxas $\mathrm{KO}$ cells (Fig. 5c and Supplementary 5e). We then examined whether depletion of BRCC36, a K63-specific DUB, leads to increased SLX4/MUS81 chromatin loading or RPA hyperphosphorylation in response to CPT. Similar to that, due to Abraxas deficiency, BRCC36 knockdown led to an increased pRPA level upon CPT treatment (Fig. 5d lane 1 and 2). Chromatin fraction analysis showed that chromatin-bound SLX4/MUS81 was also increased in BRCC36 small interfering RNA (siRNA)-treated cells (Fig. 5d lane 1 and 2). In addition, knocking down BRCC36 did not further enhance pRPA level or chromatin-bound SLX4/MUS81 in Abraxas KO cells upon CPT treatment, indicating that Abraxas and BRCC36 are in the same pathway in regulating DNA end resection (Fig. 5d lane 3 and 4). As Abraxas is critical for the DUB activity of BRCC $36^{28,29}$, the elevated level of K63-conjugation in Abraxas-deficient cells is likely due to a compromised activity of BRCC36. Therefore, Abraxas restricts SLX4/MUS81 nuclease to CPT damage sites by limiting K63-linked ubiquitin conjugation on damaged chromatin.

To further confirm that uncontrolled K63-linked ubiquitin conjugation leads to increased chromatin loading of SLX4/ MUS81 and excessive resection, we tested whether decreasing K63-linked ubiquitin conjugation in Abraxas KO cells can rescue the hyperphosphorylation of RPA in response to CPT. Knocking 
down the K63-specific E2 conjugating enzyme Ubc13 in $\mathrm{KO}$ cells decreased the amount of MUS81 on chromatin and reduced the hyperphosphorylation of RPA and chromatin-associated RPA70 levels (Fig. 5e lane 3 and 4). Reduction of RNF168, an E3 ligase involved in K63-ubiquitin conjugation, also reduced pRPA and chromatin-bound MUS81 levels in Abraxas KO cells as well (Fig. 5f and Supplementary Fig. 5f). In addition, inhibition of ATM abolished the elevated pRPA levels in Abraxas KO cells (Supplementary Fig. 5g), consistent with the previous findings that K63-linked ubiquitin modification at DSBs is dependent on ATM activation ${ }^{13,26}$.

Thus, through counteracting RNF168/Ubc13-dependent K63-linked ubiquitin conjugation, Abraxas/BRCA1-A complex restricts SLX4/MUS81 nuclease loading to CPT damage sites and limits excessive resection at CPT-induced DSBs.

Abraxas limits MiDAS via RAD52, and POLD3-dependent and
RAD51-independent BIR. Although Abraxas/BRCA1-A complex has been suggested a role in inhibiting HR in the repair of $\mathrm{DSBs}^{32-34}$, Abraxas deficiency led to a decrease of RAD51 foci in response to CPT (Supplementary Fig. 6a). It thus is intriguing how the increased resection in Abraxas-deficient cells effect on HR. It has been established in yeast that repair of seDSB is carried out by BIR pathway ${ }^{15-17}$. We thus investigated whether the excessive resection in Abraxas-deficient cells involves BIR. BIR depends on RAD52-mediated strand invasion and POLD3dependent DNA synthesis ${ }^{15-17}$. We found that knocking down RAD52 and POLD3 rescued RPA hyperphosphorylation in Abraxas $\mathrm{KO}$ cells, indicating that the excessive resection in Abraxas-deficient cells is dependent on RAD52 and POLD3 (Fig. 6a and Supplementary Fig. 6). Depletion of RAD51, however, had little effect on the hyperphosphorylation of RPA (Fig. 6b). Thus, the increased resection in Abraxas KO cells likely involves BIR-like mechanism that is independent of RAD51. Interestingly, knockdown of RAD52 and POLD3 also reduced MUS81 levels on chromatin in Abraxas KO cells (Fig. 6a). This is similar to the previous finding that RAD52 promotes recruitment of MUS81 to stalled replication forks at CFSs for $\mathrm{BIR}^{18}$. In addition, chromatin-bound RAD52 was elevated in Abraxas KO cells treated with CPT (Supplementary Fig. 6e). It is thus likely that increased resection triggers RAD52-dependent BIR, which further promotes the recruitment of MUS81 forming a positive feedback loop that leads to even more enhanced resection.

In yeast, a majority of BIR events are completed during G2/ $\mathrm{M}^{17}$. In mammals, MiDAS shares features with BIR for repair and restart of collapsed DNA replication forks at CFSs in response to replication stress ${ }^{18,19}$. We investigated whether CPT-induced increased resection in Abraxas-deficient cells is associated with MiDAS. After treatment of CPT and arrested in late G2 with a CDK1 inhibitor RO-3306, cells were released into mitosis in the presence of EdU. After collecting cells with mitotic shake-off and performing Click-it chemistry, we observed that EdU incorporation was detected in CPT-treated cells and EdU-positive mitotic cells were significantly increased in Abraxas KO cells when compared to the control (Fig. 6c). When we treated cells with RAD52 inhibitor, the increased MiDAS in Abraxas KO cells is abolished (Fig. 6d), indicating that the increased MiDAS in KO cells in response to CPT is dependent on RAD52, consistent with the established role of RAD52 in promoting BIR and MiDAS ${ }^{18,51}$. To further assess the effect of replication-associated CPT damage, we first treated cells with thymidine for $24 \mathrm{~h}$ arresting cells in G1/ $\mathrm{S}$ before release into $\mathrm{S}$ phase for $1 \mathrm{~h}$ treatment with CPT and subsequent MiDAS assay (Fig. 6e). Similarly, the percentage of cells undergoing MiDAS was much greater in $\mathrm{KO}$ cells when compared to the control (Fig. 6e). Furthermore, knocking down
MUS81 significantly reduced EdU incorporation into mitotic cells, indicating that CPT-induced MiDAS is dependent on MUS81 (Fig. 6e). This is consistent with the requirement of MUS81 for MiDAS at CFSs under replication stress ${ }^{18}$.

Together, these data indicate that Abraxas plays a critical role in suppressing CPT-induced damage repair through BIR mechanism such as MiDAS.

Abraxas deficiency leads to increased chromosome aberrations and CPT sensitivity. BIR is a mutagenic repair pathway that is associated with elevated levels of mutagenesis and chromosomal rearrangements ${ }^{15-17}$. The excessive DNA end resection and increased BIR events in Abraxas-deficient cells predicts elevated genomic aberrations in Abraxas-deficient cells. We investigated chromosome aberrations in WT and Abraxas-/- MEF cells treated with CPT using metaphase spread. We found that chromosome aberrations significantly increased in Abraxas-/MEFs, including chromosome breaks, radial structures, fusions, and multi-breaks fusions (Fig. 7a, b). Abraxas $\mathrm{KO}$ cells are sensitive to CPT as detected by colony formation assay after CPT treatment (Fig. 7c). As the excessive resection and increased MiDAS in Abraxas-deficient cells depend on MUS81, we also examined whether knockdown of MUS81 can rescue the survival of Abraxas $\mathrm{KO}$ cells in response to CPT treatment. We found that, however, MUS81 knockdown sensitized Abraxas KO cells to CPT as detected by colony formation assay (Fig. 7d) and cell viability assay (Fig. 7e). It is possible that although uncontrolled BIR leads to cell death, regulated BIR may still be a necessary mechanism for Abraxas-deficient cells to survive CPT-induced DNA damage. Alternatively, it is also possible that the role of MUS81 in processing other replication intermediates in restarting the collapsed replication forks is required for Abraxas $\mathrm{KO}$ cell survival in response to CPT.

\section{Discussion}

We have demonstrated a role of Abraxas/BRCA1-A complex in limiting excessive DNA end resection, R-loop accumulation, and cells undergoing BIR-dependent MiDAS. Through counteracting RNF168/Ubc13-dependent K63-linked ubiquitin conjugation, Abraxas restricts SLX4/MUS81 recruitment to CPT damage sites for cleavage and subsequent resection to generate ssDNA. In the absence of Abraxas, increased K63-linked ubiquitin conjugation leads to SLX4/MUS81 overloading on chromatin, generating increased seDSBs, which are further processed by MRE11 endonucleases, CTIP and DNA2/BLM, forming an extensive length of ssDNA. Uncontrolled MUS81 loading and excessive end resection also involve RAD52- and POLD3-dependent BIR that results in increased MiDAS, extensive chromosome aberrations, and cell lethality in Abraxas-deficient cell (Fig. 8).

Abraxas limits replication-associated seDSBs resection. Abraxas specifically limits end resection of replication-associated DSBs, as inhibition of DNA replication by APH completely abolished the excessive resection in Abraxas-deficient cells treated with CPT (Fig. 1). The inhibitory role of Abraxas on IR-induced DSBs end resection appears at a later time point $(4 \mathrm{~h})$ after treatment, possibly when neighboring replication forks emerge and collide into the DSBs, as inhibition of replication by APH significantly decreased the level of resection. MUS81 plays a central role in mediating the effect of Abraxas in inhibiting excessive DNA end resection. Abraxas inhibits DNA end resection of replication-associated DSBs by restraining K63-linked ubiquitin-dependent SLX4/MUS81 recruitment. SLX4 interacts with MUS81 and binds to K63-linked ubiquitin chain through its tandem UBZ domain modification ${ }^{48-50}$. Increased K63-linked 
a

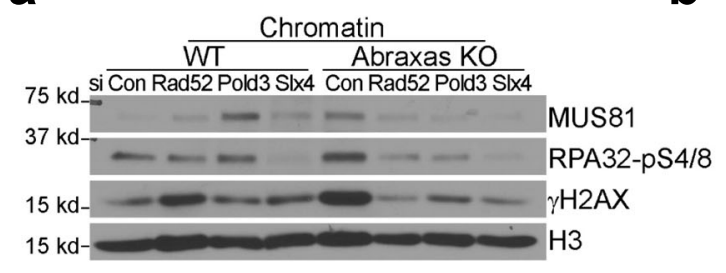

b

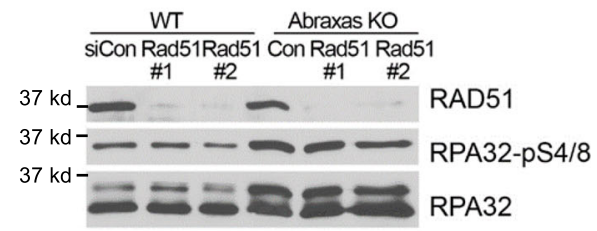

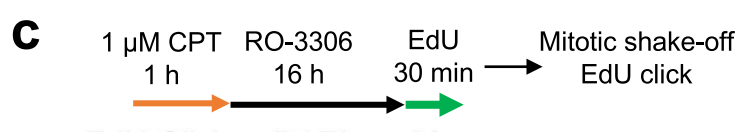

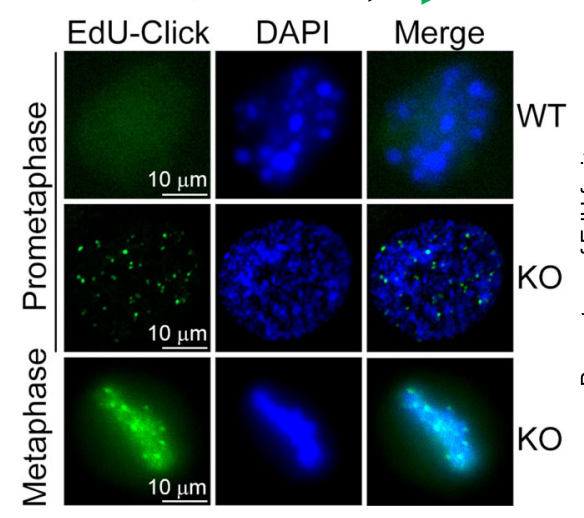

e d

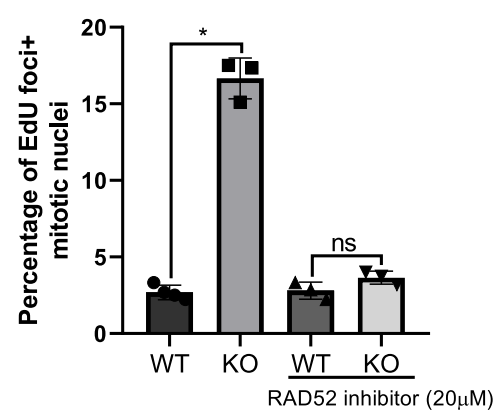

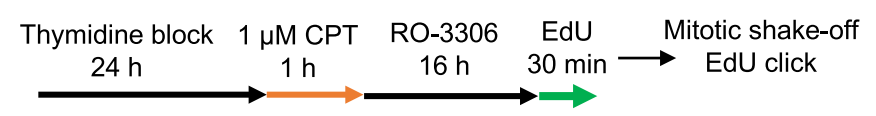



Fig. 6 Abraxas limits mitotic DNA synthesis via RAD52, and POLD3-dependent and RAD51-independent BIR. a Knocking down RAD52 or POLD3 reduced RPA hyperphosphorylation and MUS81 chromatin loading in Abraxas KO cells treated with CPT. Cells transfected with indicated siRNAs were treated with CPT. Chromatin fraction were prepared and used for western blottings. b RPA hyperphosphorylation in Abraxas KO cells treated with CPT is independent of RAD51. $\mathbf{c}$ Increased mitotic DNA synthesis in Abraxas KO cells in response to CPT. EdU-click reaction was performed to detect mitotic DNA synthesis. A representative image of EdU foci (left panel) and quantification of the percentage of EdU foci+ cells were shown as mean value \pm SD for U2OS ( $n=3$ independent experiments with total 292 cells), KO ( $n=3$ independent experiments with total 265 cells) examined (right panel). Two-tailed unpaired Student's $t$-test was used for statistical analysis. ${ }^{* \star *} p=0.0001$. d Inhibition of RAD52 reduces MiDAS in Abraxas KO cells treated with CPT. Cells were treated similarly as above. RAD52 inhibitor was added during EdU pulse labeling and quantification of the percentage of EdU foci+ cells were shown as mean value \pm SD for WT ( $n=4$ independent experiment with total 183 cells), KO ( $n=3$ independent experiment with total 167 cells), WT/RAD52 inhibitor ( $n=3$ independent experiments with total 121 cells), KO/RAD52 inhibitor ( $n=3$ independent experiments with total 109 cells) examined. ${ }^{*} p=$ 0.042. e MUS81 depletion inhibits mitotic DNA synthesis in Abraxas KO cells treated with CPT. Cells transfected with indicated siRNAs were incubated with thymidine before treatment of CPT and assay for mitotic DNA synthesis. Percentage of EdU foci+ cells were quantified and shown as mean value \pm SD for WT-siCon ( $n=9$ independent experiments with total 134 cells), KO-siCon ( $n=9$ independent experiments with total 108 cells), WT-siMUS81 ( $n=4$ independent experiments with total 172 cells), and KO-siMUS81 ( $n=4$ independent experiments with total 141 cells). One-way ANOVA Kruskal-Wallis test was used for statistical analysis. ${ }^{\star} p=0.0440$ and $p=0.0275$. See also Supplementary Fig. 6. 
a

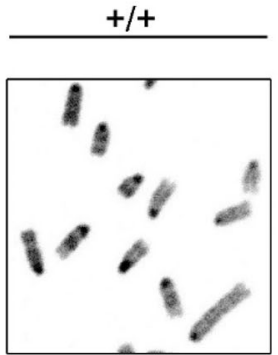

b

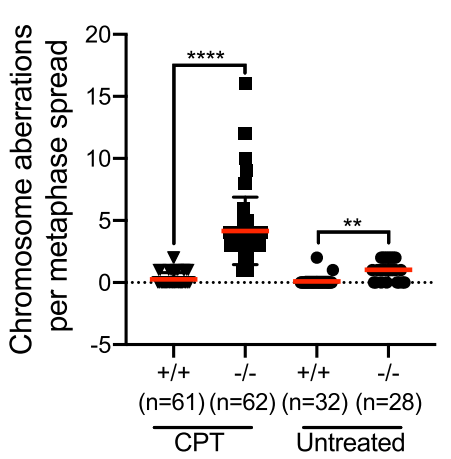

d

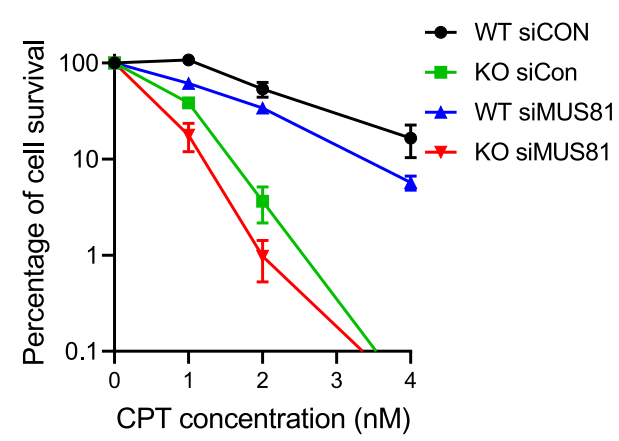

$-1-$

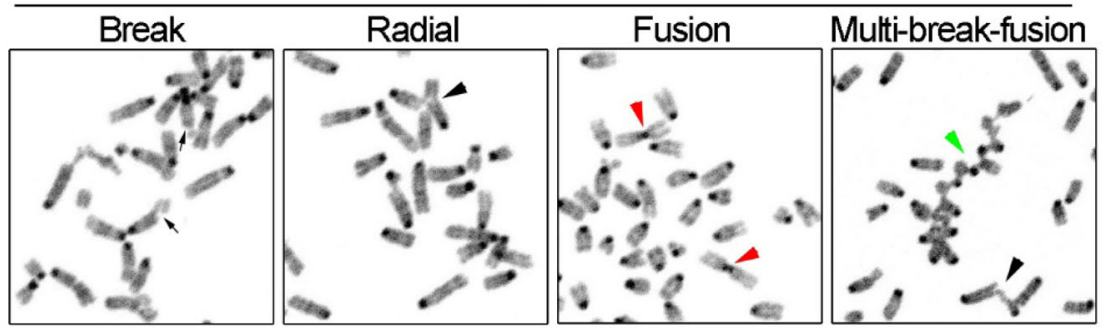

UNTR


e

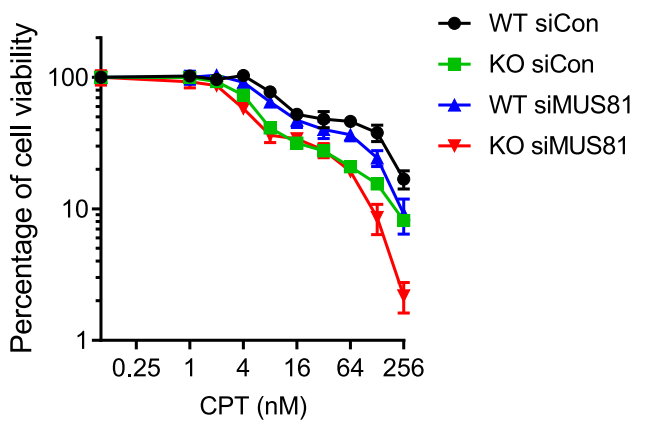

Fig. 7 Abraxas deficiency leads to increased chromosome aberrations and cellular sensitivity to CPT. a Abraxas-/- MEFs displayed increased chromosome aberrations upon CPT treatment. Metaphase spreads were prepared from immortalized MEFs untreated or treated with $1 \mu \mathrm{M}$ CPT for $1 \mathrm{~h}$. Representative images of break (black arrows), radial structure (black arrowhead), fusion (red arrowhead), and multiple breaks and fusions (green arrowhead) of cells treated with CPT are shown. $\mathbf{b}$ Quantification of chromosome aberrations in Abraxas $-/-$ and $+/+$ MEFs. " $n$ " represents the number of metaphases examined over three independent experiments. The red bar represents mean value and the error bars represent \pm SD. One-way ANOVA Kruskal-Wallis test was used for statistical analysis. ${ }^{\star \star \star \star} p<0.0001,{ }^{\star \star} p=0.0052$. c Abraxas KO U2OS cells are sensitive to CPT treatment as detected by colony formation assay. Percentage of cell survival (left) and representative images of colony formation of WT and Abraxas KO U2OS cells untreated or treated with CPT (right) are shown. Two-tailed unpaired Student's $t$-test was used for statistical analysis. $N=3$ biologically independent replicates. Data are presented as mean value \pm SD. ${ }^{\star \star \star} p=0.0003$. d CPT sensitivity detected by colony formation assay in WT and Abraxas KO U2OS cells transfected with indicated siRNA. Data are represented as mean \pm SD. $N=3$ biologically independent replicates at each CPT dose. e CPT sensitivity measured by cell viability assay. WT and Abraxas KO U2OS cells were transfected with indicated siRNA. Cells were seeded into 96 -well cell culture plate at $24 \mathrm{~h}$ after transfection and treated with serial doses of CPT for 3 days. Viability was measured and quantified using CCK-8 kit. Data are represented as mean \pm SD. $N$ $=3$ biologically independent replicates at each CPT dose.

ubiquitin conjugation on damaged chromatin due to Abraxas or BRCC36 deficiency leads to increased SLX4/MUS81 loading on damaged chromatin (Fig. 5 and Supplementary Fig. 5). As MUS81 mediates cleavage of CPT-induced stalled replication forks ${ }^{4}$, increased MUS81 chromatin loading results in elevated generation of DSBs, which are further processed by additional nucleases for DNA end resection including Mre11, CtIP, and DNA2, leading to excessive resection and generation of extended length of ssDNA in Abraxas-deficient cells. Knockdown of SLX4 or MUS81 decreases DSBs generation and eliminates the excessive resection (Figs. 3 and 5). Another piece of evidence that supports MUS81 functions upstream during the resection of DSBs ends is that knocking down downstream nucleases such as CtIP or DNA2 does not affect the amount of MUS81 on damaged chromatin but reduced excessive resection (Supplementary Fig. 5c). MUS81 is a structure-specific nuclease that cleaves replication fork-like structures in the recovery of stalled replication forks ${ }^{37,38}$. Collapsed replication forks induced by PARP1 trapping by olaparib, DNA crosslinks generated by MMC, or Rloop-impeded replication forks may possess similar structural features that are subjected to MUS81 cleavage and are regulated by Abraxas. The DNA structural determinant of the stalled 


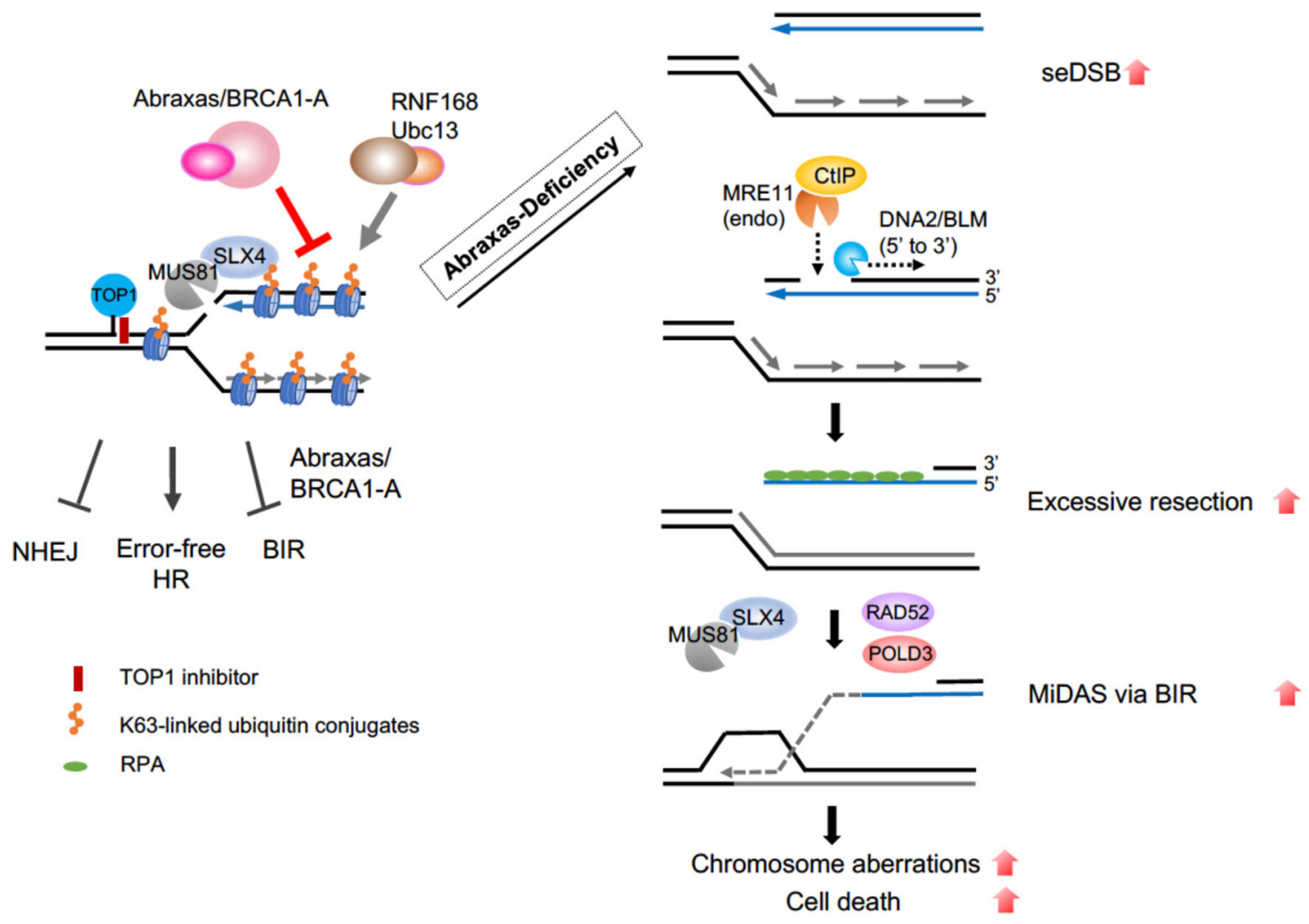

Fig. 8 A proposed model for the role of Abraxas/BRCA1-A complex. Abraxas/BRCA1-A complex inhibits DNA end resection and limits BIR by restricting SLX4/MUS81 chromatin loading through counteracting K63-linked ubiquitin modification catalyzed by RNF168/Ubc13 in response to CPT. In the absence of Abraxas, increased K63-linked ubiquitin conjugation leads to SLX4/MUS81 overloading on chromatin, generating increased seDSBs, which are further processed by MRE11 endonucleases, CTIP and DNA2/BLM, forming an extensive length of ssDNA. Uncontrolled MUS81 loading and excessive end resection also involve RAD52- and POLD3-dependent BIR that results in increased mitotic DNA synthesis, extensive chromosome aberrations, and cell lethality in Abraxas-deficient cell.

replication intermediates of MUS81-mediated cleavage still needs to be defined but is likely to be unique, as increased resection in Abraxas-deficient cells in response to CPT is independent of SMARCAL1, ZRANB3, and HLTF, factors that are needed for replication fork reversal ${ }^{39-44}$.

It is noted that the excessive resection in Abraxas-deficient cells requires MRE11 endonuclease but not exonuclease activity. As CPT-induced replication-associated DSBs are seDSBs, it suggests that the initiation of resection of seDSBs is similar to that of twoended DSBs, which is initiated by MRE11 endonuclease activity and CtIP. However, unlike the processing of two-ended DSBs, which are followed in both directions, the subsequent resection of seDSBs appears to be independent of MRE11 $3^{\prime}-5^{\prime}$ exonuclease activity such that mirin, an inhibitor of Mre11 exonuclease activity, does not decrease the resection (Fig. 4). In addition, whereas the resection is dependent on DNA2 nuclease and BLM helicase, it is independent of EXO1. For repair of seDSBs, only one broken end is available for strand invasion, which triggers BIR with DNA repair synthesis proceeding via a D-loop. It is possible that a specific complex structure arises during the progression of the resection of seDSBs that favors the use of DNA2/BLM instead of EXO1/BLM. Notably, excessive resection and increased MUS81 chromatin loading in Abraxas-deficient cells are dependent on RAD52 and POLD3, two essential factors for BIR. BIR is preceded by extensive $5^{\prime}$ - to $3^{\prime}$-end resection to generate $3^{\prime}$-ssDNA. In addition, RAD52 appears to promote recruitment of MUS81 during replication stress-induced MiDAS at CFS via BIR ${ }^{18}$. Thus, it is likely that following initial MUS81mediated cleavage, increased resection in Abraxas-deficient cells triggers the initiation of BIR and the proceeding of BIR, in turn, further re-enforces the accumulation of MUS81 and ssDNA, forming a positive feedback loop leading to MUS81 overloading, excessive end resection, and uncontrolled MiDAS.

Role of K63-linked ubiquitin modification in replicationassociated DSBs end resection. The increased recruitment of SLX4/MUS81 to chromatin in Abraxas-deficient cells is dependent on K63-linked ubiquitin modification catalyzed by RNF168 and Ubc13. As a molecular scaffold, SLX4 interacts with MUS81 and promotes MUS81 activity ${ }^{46,47}$. Our data indicate that SLX4/ MUS81 is recruited to CPT damage sites through SLX4 UBZ domains binding to K63-linked ubiquitin chain. It is also possible that both K63-linked ubiquitin modification and replication intermediate structure formed by replication-associated DSBs are determinants of the SLX4/MUS81 recruitments.

K63-linked ubiquitin modification plays an important role in the recruitment of Abraxas/BRCA1-A complex through Rap80 binding to K63-linked ubiquitin chain. K63-linked ubiquitin modification at DSBs is dependent on ATM activation; thus, inhibition of ATM in Abraxas KO cells abolished the increased resection in response to CPT (Supplementary Fig. 5). This is consistent with the recent findings that ATM-deficiency decreases DNA end resection, and that depletion of BRCA1-A complex component in ATM-deficient cells restores DNA end resection at topotecan damaged DNA ${ }^{32}$. Abraxas/BRCA1-A complex, the recruitment of which depends on K63-linked ubiquitin modification, in turn, through its DUB activity, counteracts the activity of RNF168/Ubc13 in K63-linked ubiquitin conjugation, thus keeping the balance of K63-ubiquitin modification at DSBs. This balance is likely to be critical in controlling the levels of SLX4/ 
MUS81 at DSBs. In the absence of Abraxas or BRCC36, elevated levels of K63-conjugation leads to increased chromatin loading of SLX4/MUS81 resulting in excessive end resection at replicationassociated DSBs. Our study thus highlights the importance of the balance of K63-dependent ubiquitin modification at DSBs in finetuning the degree of resection by controlling the amount of chromatin-bound SLX4/MUS81 in response to CPT.

Abraxas/BRCA1-A complex inhibits BIR. Decades of studies in yeast have indicated that repair of seDSBs resulted from replication fork breakage is carried out by BIR pathway ${ }^{15,17}$. BIR is a specific form of HR that is employed to restore the replication fork by using the sister chromatid as template. The regulation of BIR in mammalian cells, however, is less clear. RAD52- and POLD3-dependent, RAD51-independent MiDAS occurring at CFS in response to replication stress shares features with BIR and its initiation is promoted by cleavage of stalled replication forks at fragile sites by SLX4/MUS8118,19,51. Our studies show that, similarly, CPT-induced extensive resection that triggers MiDAS in Abraxas-deficient cells is mediated by SLX4/MUS81 cleavage and depends on RAD52 and POLD3, but not on RAD51. It is possible that the structure features associated with collapsed replication forks at CFSs leading to MiDAS are similar to those resulted by CPT-induced fork collapse, triggering RAD51independent BIR.

BIR involves extensive DNA resection and mutagenic DNA synthesis, and is a mutagenic pathway, which when uncontrolled, can lead to cell death. How cells limit the usage of BIR in repair of DSBs is not well understood. Our studies propose that Abraxas/ BRCA1-A complex plays a crucial role in restricting cells undergoing BIR in the repair of replication-associated seDSBs. The increased cellular sensitivity of Abraxas KO cells to CPT is likely due to both of the increased DSBs and the uncontrolled increase of BIR due to Abraxas deficiency. At sites of collapsed replication forks as seDSBs arise, precise resection at the break end is likely important not only to prevent NHEJ through mechanisms such as removal of $\mathrm{Ku}^{52}$, it is also critical in inhibiting cells undergo mutagenic HR pathway such as BIR. The K63-linked ubiquitin modification at damaged sites likely plays a crucial role in determining the precision of pathway choice and needs to be fine-tuned through balancing with the Abraxas/ BRCA1-A complex DUB activity. We propose that, in addition to the choice of NHEJ and HR, a regulatory mechanism through regulating SLX4/MUS81 chromatin loading by Abraxas/BRCA1A complex exists to control the choice of BIR and other types of HR.

Together, our study provides mechanistic insights into the role of Abraxas/BRCA1-A complex in inhibiting mutagenic repair mechanism BIR at replication-associated seDSBs to protect genome stability.

\section{Methods}

Cell line and cell culture. U2OS cells were grown in McCoy's 5A with L-glutamine medium (Cellgro, Corning) supplemented with 10\% fetal bovine serum (FBS) (Gibco) and $1 \%$ penicillin/streptomycin (Gibco). Abraxas KO U2OS cells were generated by using CRISPR-Cas9 as described previously ${ }^{31}$. 293 T cell line was grown in Dulbecco's modified Eagle's medium (DMEM) (Cellgro, Corning) with $4.5 \mathrm{~g} / \mathrm{L}$ glucose, $\mathrm{L}$-glutamine, and sodium pyruvate medium supplemented with $10 \%$ FBS and $1 \%$ penicillin/streptomycin. Immortalized MEFs were cultured in DMEM with $4.5 \mathrm{~g} / \mathrm{L}$ glucose, L-glutamine, and sodium pyruvate medium supplemented with $10 \%$ FBS and $1 \%$ penicillin/streptomycin.

Chemicals, plasmids, siRNAs, and antibodies. Chemicals used in this study including CPT (Sigma, C9911), MMC (Sigma, M4287), Hydroxyurea (HU, Sigma, H8627), BrdU (Sigma, B5002), 5, 6-dichloro-1- $\beta$-d-ribofuranosylbenzimidazole (DRB, Sigma, D1916), a-Amanitin (Sigma, A2263), APH (Sigma, A0781), Thymidine (Sigma, T1895), RO-3306 (Sigma, SML0569), VE-821 (Sigma, SML1415), Mirin (Sigma, M9948), KU55933 (Selleckchem, S1092), Olaparib (Selleckchem,
S1060), YOYO-1 (Invitrogen, Y3601), AICAR (RAD52 inhibitor, Sigma A9978), and PFM01 and PFM03 (from Dr. John A. Tainer).

The pENTR-RNASEH1 plasmid was purchased from MDACC shRNA and ORFeome Core in MDA Anderson and was inserted into MSCV-HA retroviral expression vector by performing Gateway Recombination Cloning Technology using LR clonase (Invitrogen). HA-tagged SLX4 WT, UBZ, and DM mutants expression plasmids were a gift from Dr. Lee Zou ${ }^{50}$. Expression constructs of Abraxas WT and mutants were generated previously ${ }^{31}$. SLX4 siRNA\#1: 5'-AAA CGTGAATGAAGCAGAA-3', siRNA\#2: 5'-CGGCATTTGAGTCTGCAGGT GAA-3', and siRNA-UTR7062: 5'-GCACCAGGTTCATATGTAT-3'; MUS81 siRNA\# 1: 5'-CAGCCCTGGTGGATCGATA- $3^{\prime}$ and siRNA\#2: 5'-CATTAAGTGT GGGCGTCTA-3'; DNA2 siRNA\#1: 5'-CAGTATCTCCTCTAGCTAG-3', siRNA\#2: 5'-ATAGCCAGTAGTATTCGAT-3', DNA2 siRNA\#3: 5'-AGACAAGG UUCCAGCGCCA-3'; BLM siRNA\#1: 5'-AGCAGCGATGTGATTTGCA- $3^{\prime}$ and siRNA\#2: 5'-ATCAGCTAGAGGCGATCAA-3'; RAD51 siRNA\#1: 5'-GAGCT TGACAAACTACTTC- $3^{\prime}$ and siRNA\#2: 5'-GACTGCCAGGATAAAGCTT-3'; RAD52 siRNA\#1: 5'-GGCATTATGTCTAGGACTA-3' and siRNA\#2: 5'-CAAT TAGTGGTTAGGGAAA-3'; SMARCAL1 siRNA\# 1: 5'-GCTTTGACCTTCTT AGCAA-3' ${ }^{\prime}$, siRNA\#2: 5'-AAGCAAGGCCCATCCCAAA-3' , and siRNA\#3: 5'-CA CCCTTTGCTAACCCAACTCATAA-3'; HLTF siRNA: 5'-GGAATATAATG TTAACGAT-3'; POLD3 siRNA: 5'-CAACAAGGCACCAGGGAAA-3'; and ZRANB3 siRNA 5'-GAGATATCATCGATTATGA-3'12. ON-TARGETplus human siRNA smart pool for EXO1 (L-013120-00-0005), MRE11 (L-009271-000005), and ON-TARGETplus Non-targeting siRNA (D-001810-01-20) were purchased from Dharmacon. BRCA1, CTIP, BRCC36, Abraxas, UBC13, and RNF8 siRNAs were purchased from Invitrogen as described previously ${ }^{23,24}$. siRNAs were transfected using Lipofectamine RNAiMAX (Invitrogen).

Antibodies used for immunoblotting include MUS81 (Abcam, ab14387, $1: 1000$ dilution), Abraxas (homemade, $1: 1000$ dilution) ${ }^{23}$, SLX4 (Bethyl Laboratories, A302-269A, $1: 1000$ dilution), HA (Cell Signaling Technology, 3724s and 2367s, $1: 1000$ dilution), K63 (EMD Millipore, 05-1308, $1: 3000$ dilution), RPA32-pS4/8 (Bethyl Laboratories, A300-245A, $1: 2000$ dilution), RPA32 (Bethyl Laboratories, A300-244A, $1: 2000$ dilution), RPA70 (Bethyl Laboratories, A300-241A, $1: 1000$ dilution), p-Chk1 S317 (Cell Signaling Technology, 2344, 1: 500 dilution), p-Chk2 Thr68 (Cell Signaling Technology, 2661, $1: 1000$ dilution), Chk1 (Santa Cruz, sc8408, $1: 100$ dilution), $\gamma \mathrm{H} 2 \mathrm{AX}$ (Millipore, 05-636, $1: 1000$ dilution), DNA2 (Abcam, ab96488, 1:500 dilution), BLM (Bethyl Laboratories, A300-110A, 1:1000 dilution), EXO1 (Millipore, ABE1354, 1 : 1000 dilution), BRCC36 (Bethyl Laboratories, IHC-00715, $1: 1000$ dilution), RAD51 (Calbiochem, PC130, $1: 1000$ dilution), RAD52 (Santa Cruz, sc-365341, $1: 100$ dilution), POLD3 (Bethyl Laboratories, A301-244A, 1 : 1000 dilution), SMARCAL1 (Santa Cruz, sc-376377, $1: 100$ dilution), HLTF (Santa Cruz, sc-398357, $1: 100$ dilution), Ubc13 (Zymed, 37-1100, 1:500 dilution), H3 (Abcam, ab1791, $1: 1000$ dilution), Lamin A (Sigma, L1293, $1: 1000$ dilution), CtIP (Cell Signaling Technology, 9201, $1: 1000$ dilution) RNF168 (Millipore, ABE367, 1:500 dilution), and GAPDH (Invitrogen, MA515738, 1:5000 dilution). Antibodies used for IF include RPA32-pS4/8 (Bethyl Laboratories, A300-245A, 1:1000 dilution), BrdU (BD Bioscience, 347580, 1:500 dilution), $\gamma \mathrm{H} 2 \mathrm{AX}$ (Millipore, 05-636, $1: 1000$ dilution), MUS81 (Abcam, ab14387, $1: 200$ dilution), MUS81 (Santa Cruz, sc-53382, $1: 100$ dilution), RAD51 (Calbiochem, PC130, 1:500 dilution), MRE11 (Novus, NB100-142, 1:1000 dilution), and S9.6 (kerafast, ENH001, 1:500 dilution).

Immunoblotting and chromatin fractionation. Cells were lysed using NETN buffer (50 mM Tris- $\mathrm{HCl} \mathrm{pH} 8.0,150 \mathrm{mM}$ sodium chloride, $1 \mathrm{mM}$ EDTA, 0.5\% Nonidet P-40, $1 \mathrm{mM}$ dithiothreitol (DTT), $1 \mathrm{mM}$ phenylmethylsulfonyl fluoride, 5 $\mathrm{mM} \mathrm{NaF}, 1 \mathrm{mM} \mathrm{Na} \mathrm{VO}_{4}, 50 \mathrm{mM} \beta$ - glycerophosphate, and protease inhibitor cocktail), sonicated, and centrifuged at $4{ }^{\circ} \mathrm{C}$ using $21,000 \times g$ for $10 \mathrm{~min}$. The supernatant was collected and used as total cell lysate. For chromatin fractionation, $2 \times 10^{6}$ cells were incubated with $300 \mu$ cold buffer A (10 mM HEPES pH 7.9, $10 \mathrm{mM} \mathrm{KCl}, 1.5 \mathrm{mM} \mathrm{MgCl}_{2}, 0.34 \mathrm{M}$ sucrose, $10 \%$ glycerol, $5 \mathrm{mM} \mathrm{NaF}, 1 \mathrm{mM}$ $\mathrm{Na}_{3} \mathrm{VO}_{4}, 1 \mathrm{mM}$ DTT, protease cocktail, and $0.2 \%$ Triton X-100) for $5 \mathrm{~min}$ on ice. After centrifugation at $1500 \times g$ at $4{ }^{\circ} \mathrm{C}$ for $5 \mathrm{~min}$, cell pellets were collected and washed with cold buffer A. Cell pellets were then incubated with cold buffer B ( $3 \mathrm{mM}$ EDTA, $0.2 \mathrm{mM}$ EGTA, $1 \mathrm{mM}$ DTT, and protease inhibitor cocktail) for 30 min on ice. After centrifugation, pellets were collected and washed with buffer B, then resuspended in NETN buffer. After sonication and centrifugation at $4{ }^{\circ} \mathrm{C}$ using $21,000 \times g$ for $10 \mathrm{~min}$, the supernatant was collected and used as chromatin fraction.

Immunofluorescence. Cells grown on coverslips were fixed with freshly made 3\% paraformaldehyde/2\% sucrose solution for $10 \mathrm{~min}$ after CPT treatment. For chromatin-bound MUS81, RAP32-pS4/8, and S9.6 staining, cells were preextracted before fixation using pre-extraction buffer $(10 \mathrm{mM}$ PIPES $\mathrm{pH} 6.8$, $100 \mathrm{mM}$ sodium chloride, $300 \mathrm{mM}$ sucrose, $3 \mathrm{mM}$ magnesium chloride, $1 \mathrm{mM}$ EGTA, $0.2 \%$ Triton X-100). After fixation, cells were permeabilized with $0.5 \%$ Triton X-100 solution for $5 \mathrm{~min}$ on ice, washed with phosphate-buffered saline (PBS), and stained with indicated primary antibodies diluted in $1 \%$ bovine serum albumin (BSA) at $37^{\circ} \mathrm{C}$ for $1 \mathrm{~h}$ followed by incubation with secondary antibodies conjugated with Alexa-488 (Invitrogen, A11008, 1:1000 dilution) or Alexa-555 (Invitrogen, A31570, 1:1000 dilution) for $1 \mathrm{~h}$ at $37^{\circ} \mathrm{C}$. Coverslips were then 
washed with PBS and mounted using 4',6-diamidino-2-phenylindole (DAPI) containing antifade solution (Invitrogen). For cells pulse-labeled with EdU $(10 \mu \mathrm{M})$ and IF staining, cells were fixed, permeabilized, and stained with indicated primary and secondary antibodies, followed by EdU-click reaction using Click-iT EdU Alexa Fluor 488 imaging kit. Images were collected with 80i eclipse Nikon microscope using $\times 40$ or $\times 63$ objective using NIH Elements AR software (AR 5.10 .0164 bit software).

Native BrdU staining. Cells were labeled with BrdU $(10 \mu \mathrm{M})$ for $36 \mathrm{~h}^{53}$, washed with culture medium after labeling, and treated with CPT $(1 \mu \mathrm{M}, 1 \mathrm{~h})$. Cells were then pre-extracted with pre-extraction buffer, fixed with $3 \%$ paraformaldehyde $/ 2 \%$ sucrose solution, treated with cold methanol for $20 \mathrm{~min}$ at $-20^{\circ} \mathrm{C}$, washed with PBS, and treated with cold acetone for $30 \mathrm{~s}$. After blocking with $2 \%$ BSA for $1 \mathrm{~h}$, staining was performed with BrdU antibody followed by Alexa-488-conjugated secondary antibody. Images were captured with $80 \mathrm{i}$ eclipse Nikon microscope using $\times 40$ objective. BrdU nuclear intensity was measured by ImageJ software (ImageJ 2.0 and 64 bit Java8 from https://imagej.nih.gov).

S9.6 Dot Blot. Genomic DNA was extracted from cells using DNeasy Blood \& Tissue Kit (Qiagen, 69504) following the manufacturer's instruction. Genomic DNA $(500 \mu \mathrm{g})$ of each sample was blotted onto positively charged nylon membrane in duplication using dot blot apparatus. After UV crosslink at $0.12 \mathrm{~J} / \mathrm{cm}^{2}$ using UV Stratalinker 1800 for twice, the membrane was blocked in 5\% milk/TBST buffer (20 mM Tris, $150 \mathrm{mM} \mathrm{NaCl}, 0 / 1 \%$ Tween-20), and then incubated with 59.6 antibody, followed by incubation with secondary antibody. The membrane was then reused, stripped, washed, and stained with SYBR Gold (Thermal Fisher, S11494). Image was captured using ChemiDoc imaging system (Bio-Rad).

Neutral comet assay. Cells were trypsinized, collected, and resuspended in PBS at a concentration of 100,000 cells $/ \mathrm{ml}$. Ten microliters cell suspension (10,000 cells) was mixed with $100 \mu \mathrm{l}$ low-melting agarose gel and spotted onto frosted glass slides coated with agarose gel, solidified at $4{ }^{\circ} \mathrm{C}$ in the dark. Slides were immersed into pre-chilled lysis buffer (4250-050-01, Trevigen) at $4{ }^{\circ} \mathrm{C}$ in the dark for $1 \mathrm{~h}$, to lyse the cells embedded in agarose gel. After cell lysis, slides were used for electrophoresis in neural electrophoresis (Trevigen) buffer at $4{ }^{\circ} \mathrm{C}$ in the dark with a voltage at 0.6 volt $/ \mathrm{cm}$. Slides were fixed in $70 \%$ ethanol, stained with SYBR Gold, and imaged with $80 \mathrm{i}$ eclipse Nikon microscope using $\times 10$ objective. Images were analyzed by OpenComet software (OpenComet v1.3.1 from https://cometbio.org).

Metaphase spread. Immortalized MEF cells at $60-70 \%$ confluency were treated with $1 \mu \mathrm{M}$ CPT for $1 \mathrm{~h}$ followed by incubation in medium containing $0.1 \mu \mathrm{g} / \mathrm{ml}$ colcemid (15210040) for $3 \mathrm{~h}$. Cells were then trypsinized, collected, and incubated in $0.075 \mathrm{M} \mathrm{KCl}$ hypotonic solution, then fixed with $3: 1$ methanol : acetic acid fixative. Metaphase spreads were dropped and dried on microscopy cover slides, and stained with DAPI. Images were captured by Nikon confocal microscopy.

MiDAS labeling and detection. MiDAS labeling and detection is carried out following procedures described ${ }^{19,54}$. Cells were seeded the night before to reach $80 \%$ confluency at the time when they were treated with $1 \mu \mathrm{M}$ CPT for $1 \mathrm{~h}$. Cells were then washed with fresh medium and incubated in medium with an addition of RO-3306 $(9 \mu \mathrm{M})$ for $16 \mathrm{~h}$. Cells were then washed with warm PBS and incubated in EdU $(20 \mu \mathrm{M})$-containing medium for $30 \mathrm{~min}$ for labeling. Mitotic cells were collected by shake-off, washed, and re-seeded to poly-lysine-coated coverslips. Cells were then fixed with $3 \%$ paraformaldehyde $/ 2 \%$ sucrose solution and permeabilized with $0.5 \%$ Triton X-100 solution. EdU-click reaction was performed using Click-iT EdU Alexa Fluor 488 imaging kit (C10377) following the manufacturer's instruction. Images were captured by Nikon confocal microscope. For thymidine synchronization, cells with $60-70 \%$ confluency were treated with $2 \mathrm{mM}$ thymidine for $24 \mathrm{~h}$. After wash, cells were treated with $1 \mu \mathrm{M} \mathrm{CPT}$ for $1 \mathrm{~h}$, washed with warm PBS $\left(37^{\circ} \mathrm{C}\right)$ for three times, then released into RO-3306 $(9 \mu \mathrm{M})$-containing medium for $16 \mathrm{~h}$, followed by EdU labeling and Click-iT chemistry.

Colony survival assay. Cells were transfected with indicated siRNAs. Forty-eight hours after transfection, cells were plated at low density and incubated with indicated doses of $\mathrm{CPT}$, or untreated, at $37^{\circ} \mathrm{C}, 5 \% \mathrm{CO}_{2}$ incubator for about $10-14$ days. Colonies were fixed and stained with $0.5 \%$ crystal violet $/ 20 \%$ methanol solution. Colony that has more than 50 cells was counted as a positive. Colony formation efficiency was normalized to untreated samples for the calculation of percentage cell survival. Error bars represent SD across triplicates.

Cell viability assay. Cells were transfected with siRNAs and seeded at a density of 2000 cells per well into 96 -well cell culture plate at $48 \mathrm{~h}$ after transfection. Cells were than treated with different doses of CPT continuously or left untreated for 3 days. After CPT treatment, cell viability was quantified using CCK-8 kit according to the manufacturer's instruction (Dojindo, CK04-13) and normalization to samples without treatment.
SMART assay. SMART assay was performed as described ${ }^{35}$. Cells were incubated with $\operatorname{BrdU}(10 \mu \mathrm{M})$ for $24 \mathrm{~h}$ before treatment with CPT. DNA fiber stretching in silanized coverslip was performed using Fiber $\mathrm{Comb}^{\circledast}$ molecular combing system (Genomic Vision). Coverslips containing DNA fiber were used for YOYO-1 staining or native BrdU staining. Images were captured using Nikon AI confocal microscope and analyzed using ImageJ software (ImageJ 2.0 and 64 bit Java8 from https://imagej.nih.gov).

Real-time PCR. Total RNA was extracted using RNeasy RNA extraction kit (Qiagen) and reverse transcribed using iScript cDNA synthesis kit (Bio-Rad). Realtime PCR was performed by using SYBR green supermix (Bio-Rad) and CFX-96 real-time PCR detection system (Bio-Rad). The primers used are as follows: ZRANB3 $5^{\prime}$-TCCCAGAGCTAAGTCCAGAAG- $3^{\prime}$ and $5^{\prime}$-GCATCTGCGGTT AAGAGACCATA- $3^{\prime}$; PPIA 5'-CAGACAAGGTCCCAAAGACAG-3' and 5'TCACCACCCTGACACATAAAC- $3^{\prime}$. PPIA is used as an internal control. Primers used are listed in Supplementary Table 1.

Statistics and reproducibility. All data were plotted as mean value with variances as SD using GraphPad Prism 8 software. Two-tailed Student's $t$-test was used for statistical analysis of comparison of two samples. One-way analysis of variance test was used for statistical analysis of comparisons of multiple groups. All experiments were repeated at least three times. Similar results were obtained.

Reporting summary. Further information on research design is available in the Nature Research Reporting Summary linked to this article.

\section{Data availability}

All relevant data are available from the authors upon reasonable request. Source data are provided with this paper.

Received: 9 October 2020; Accepted: 28 June 2021; Published online: 16 July 2021

\section{References}

1. Pommier, Y., Leo, E., Zhang, H. \& Marchand, C. DNA topoisomerases and their poisoning by anticancer and antibacterial drugs. Chem. Biol. 17, 421-433 (2010).

2. Pommier, Y., Sun, Y., Huang, S. N. \& Nitiss, J. L. Roles of eukaryotic topoisomerases in transcription, replication and genomic stability. Nat. Rev. Mol. Cell Biol. 17, 703-721 (2016).

3. Pommier, Y. Topoisomerase I inhibitors: camptothecins and beyond. Nat. Rev. Cancer 6, 789-802 (2006).

4. Regairaz, M. et al. Mus81-mediated DNA cleavage resolves replication forks stalled by topoisomerase I-DNA complexes. J. Cell Biol. 195, 739-749 (2011).

5. Aguilera, A. \& Garcia-Muse, T. R loops: from transcription byproducts to threats to genome stability. Mol. Cell 46, 115-124 (2012).

6. Aguilera, A. \& Gomez-Gonzalez, B. DNA-RNA hybrids: the risks of DNA breakage during transcription. Nat. Struct. Mol. Biol. 24, 439-443 (2017).

7. Sollier, J. \& Cimprich, K. A. Breaking bad: R-loops and genome integrity. Trends Cell Biol. 25, 514-522 (2015).

8. El Hage, A., French, S. L., Beyer, A. L. \& Tollervey, D. Loss of Topoisomerase I leads to R-loop-mediated transcriptional blocks during ribosomal RNA synthesis. Genes Dev. 24, 1546-1558 (2010).

9. Manzo, S. G. et al. DNA topoisomerase I differentially modulates R-loops across the human genome. Genome Biol. 19, 100 (2018).

10. Cristini, A. et al. Dual processing of R-loops and topoisomerase I induces transcription-dependent DNA double-strand breaks. Cell Rep. 28, 3167-3181 (2019). e3166.

11. Sordet, O. et al. Ataxia telangiectasia mutated activation by transcription- and topoisomerase I-induced DNA double-strand breaks. EMBO Rep. 10, 887-893 (2009).

12. Matos, D. A. et al. ATR protects the genome against $\mathrm{R}$ loops through a MUS81-triggered feedback loop. Mol. Cell 77, 514-527 (2020). e514.

13. Ciccia, A. \& Elledge, S. J. The DNA damage response: making it safe to play with knives. Mol. Cell 40, 179-204 (2010).

14. Ranjha, L., Howard, S. M. \& Cejka, P. Main steps in DNA double-strand break repair: an introduction to homologous recombination and related processes. Chromosoma 127, 187-214 (2018).

15. Llorente, B., Smith, C. E. \& Symington, L. S. Break-induced replication: what is it and what is it for? Cell Cycle 7, 859-864 (2008).

16. Anand, R. P., Lovett, S. T. \& Haber, J. E. Break-induced DNA replication. Cold Spring Harb. Perspect. Biol. 5, a010397 (2013). 
17. Kramara, J., Osia, B. \& Malkova, A. Break-induced replication: the where, the why, and the how. Trends Genet. 34, 518-531 (2018).

18. Bhowmick, R., Minocherhomji, S. \& Hickson, I. D. RAD52 facilitates mitotic DNA synthesis following replication stress. Mol. Cell 64, 1117-1126 (2016).

19. Minocherhomji, S. et al. Replication stress activates DNA repair synthesis in mitosis. Nature 528, 286-290 (2015).

20. Costantino, L. et al. Break-induced replication repair of damaged forks induces genomic duplications in human cells. Science 343, 88-91 (2014).

21. Wang, B. BRCA1 tumor suppressor network: focusing on its tail. Cell Biosci. 2 , 6 (2012).

22. $\mathrm{Hu}, \mathrm{X}$. et al. NBA1/MERIT40 and BRE interaction is required for the integrity of two distinct deubiquitinating enzyme BRCC36-containing complexes. J. Biol. Chem. 286, 11734-11745 (2011).

23. Wang, B. et al. Abraxas and RAP 80 form a BRCA1 protein complex required for the DNA damage response. Science 316, 1194-1198 (2007).

24. Wang, B. \& Elledge, S. J. Ubc13/Rnf8 ubiquitin ligases control foci formation of the Rap80/Abraxas/Brca1/Brcc36 complex in response to DNA damage. Proc. Natl Acad. Sci. USA 104, 20759-20763 (2007).

25. Hu, X., Paul, A. \& Wang, B. Rap80 protein recruitment to DNA double-strand breaks requires binding to both small ubiquitin-like modifier (SUMO) and ubiquitin conjugates. J. Biol. Chem. 287, 25510-25519 (2012).

26. Jackson, S. P. \& Durocher, D. Regulation of DNA damage responses by ubiquitin and SUMO. Mol. Cell 49, 795-807 (2013).

27. Harper, J. W. \& Elledge, S. J. The DNA damage response: ten years after. Mol. Cell 28, 739-745 (2007).

28. Feng, L., Wang, J. \& Chen, J. The Lys63-specific deubiquitinating enzyme BRCC36 is regulated by two scaffold proteins localizing in different subcellular compartments. J. Biol. Chem. 285, 30982-30988 (2010).

29. Patterson-Fortin, J., Shao, G., Bretscher, H., Messick, T. E. \& Greenberg, R. A. Differential regulation of JAMM domain deubiquitinating enzyme activity within the RAP80 complex. J. Biol. Chem. 285, 30971-30981 (2010).

30. Castillo, A. et al. The BRCA1-interacting protein Abraxas is required for genomic stability and tumor suppression. Cell Rep. 8, 807-817 (2014).

31. Wu, Q. et al. Structure of BRCA1-BRCT/Abraxas complex reveals phosphorylation-dependent BRCT dimerization at DNA damage sites. Mol. Cell 61, 434-448 (2016).

32. Balmus, G. et al. ATM orchestrates the DNA-damage response to counter toxic non-homologous end-joining at broken replication forks. Nat. Commun. 10, 87 (2019).

33. $\mathrm{Hu}, \mathrm{Y}$. et al. RAP80-directed tuning of BRCA1 homologous recombination function at ionizing radiation-induced nuclear foci. Genes Dev. 25, 685-700 (2011).

34. Coleman, K. A. \& Greenberg, R. A. The BRCA1-RAP80 complex regulates DNA repair mechanism utilization by restricting end resection. J. Biol. Chem. 286, 13669-13680 (2011).

35. Huertas, P. \& Cruz-Garcia, A. Single molecule analysis of resection tracks. Methods Mol. Biol. 1672, 147-154 (2018).

36. Cerritelli, S. M. \& Crouch, R. J. Ribonuclease H: the enzymes in eukaryotes. FEBS J. 276, 1494-1505 (2009).

37. Pepe, A. \& West, S. C. Substrate specificity of the MUS81-EME2 structure selective endonuclease. Nucleic Acids Res. 42, 3833-3845 (2014).

38. Amangyeld, T., Shin, Y. K., Lee, M., Kwon, B. \& Seo, Y. S. Human MUS81EME2 can cleave a variety of DNA structures including intact Holliday junction and nicked duplex. Nucleic Acids Res. 42, 5846-5862 (2014).

39. Betous, R. et al. SMARCAL1 catalyzes fork regression and Holliday junction migration to maintain genome stability during DNA replication. Genes Dev. 26, 151-162 (2012)

40. Ciccia, A. et al. Polyubiquitinated PCNA recruits the ZRANB3 translocase to maintain genomic integrity after replication stress. Mol. Cell 47, 396-409 (2012).

41. Kolinjivadi, A. M. et al. Smarcall-mediated fork reversal triggers Mre11dependent degradation of nascent DNA in the absence of Brca2 and stable Rad51 nucleofilaments. Mol. Cell 67, 867-881 (2017). e867.

42. Taglialatela, A. et al. Restoration of replication fork stability in BRCA1- and BRCA2-deficient cells by inactivation of SNF2-family fork remodelers. Mol. Cell 68, 414-430 (2017). e418.

43. Vujanovic, M. et al. Replication fork slowing and reversal upon DNA damage require PCNA polyubiquitination and ZRANB3 DNA translocase activity. Mol. Cell 67, 882-890 (2017). e885.

44. Bai, G. et al. HLTF promotes fork reversal, limiting replication stress resistance and preventing multiple mechanisms of unrestrained DNA synthesis. Mol. Cell 78, 1237-1251 (2020). e1237.
45. Shibata, A. et al. DNA double-strand break repair pathway choice is directed by distinct MRE11 nuclease activities. Mol. Cell 53, 7-18 (2014).

46. Svendsen, J. M. et al. Mammalian BTBD12/SLX4 assembles a Holliday junction resolvase and is required for DNA repair. Cell 138, 63-77 (2009).

47. Munoz, I. M. et al. Coordination of structure-specific nucleases by human SLX4/BTBD12 is required for DNA repair. Mol. Cell 35, 116-127 (2009).

48. Kim, Y. et al. Mutations of the SLX4 gene in Fanconi anemia. Nat. Genet. 43, 142-146 (2011).

49. Lachaud, C. et al. Distinct functional roles for the two SLX4 ubiquitin-binding UBZ domains mutated in Fanconi anemia. J. Cell Sci. 127, 2811-2817 (2014).

50. Ouyang, J. et al. Noncovalent interactions with SUMO and ubiquitin orchestrate distinct functions of the SLX4 complex in genome maintenance. Mol. Cell 57, 108-122 (2015).

51. Sotiriou, S. K. et al. Mammalian RAD52 functions in break-induced replication repair of collapsed DNA replication forks. Mol. Cell 64, 1127-1134 (2016).

52. Chanut, P., Britton, S., Coates, J., Jackson, S. P. \& Calsou, P. Coordinated nuclease activities counteract $\mathrm{Ku}$ at single-ended DNA double-strand breaks. Nat. Commun. 7, 12889 (2016)

53. $\mathrm{Xu}$, S. et al. Abrol maintains genome stability and limits replication stress by protecting replication fork stability. Genes Dev. 31, 1469-1482 (2017).

54. Min, J., Wright, W. E. \& Shay, J. W. Alternative lengthening of telomeres mediated by mitotic DNA synthesis engages break-induced replication processes. Mol. Cell Biol. 37, e00226-17 (2017).

\section{Acknowledgements}

We thank Dr. Adriana Paulucci and Department of Genetics Microscope Core Facility for assistance of imaging acquisition, and Dr. Lee Zou and Dr. John A. Tainer for reagents. This work was supported by the NIH (CA155025 to B.W.). X.W. and B.W. are also supported by NIH grant CA248088 and Cancer Prevention Research Institute of Texas (CPRIT) grant RP180244.

\section{Author contributions}

X.W. and B.W. designed the study and wrote the manuscript. B.W. supervised the project and X.W. performed the experiments.

\section{Competing interests}

The authors declare no competing interests.

\section{Additional information}

Supplementary information The online version contains supplementary material available at https://doi.org/10.1038/s41467-021-24665-w.

Correspondence and requests for materials should be addressed to B.W.

Peer review information Nature Communications thanks the anonymous reviewer(s) for their contribution to the peer review of this work.

Reprints and permission information is available at http://www.nature.com/reprints

Publisher's note Springer Nature remains neutral with regard to jurisdictional claims in published maps and institutional affiliations.

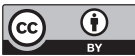

Open Access This article is licensed under a Creative Commons Attribution 4.0 International License, which permits use, sharing, adaptation, distribution and reproduction in any medium or format, as long as you give appropriate credit to the original author(s) and the source, provide a link to the Creative Commons license, and indicate if changes were made. The images or other third party material in this article are included in the article's Creative Commons license, unless indicated otherwise in a credit line to the material. If material is not included in the article's Creative Commons license and your intended use is not permitted by statutory regulation or exceeds the permitted use, you will need to obtain permission directly from the copyright holder. To view a copy of this license, visit http://creativecommons.org/ licenses/by/4.0/.

(c) The Author(s) 2021 